\title{
Invariant States and Conditional Expectations of the Anticommutation Relations
}

\author{
John C. Wolfe ${ }^{\star}$ \\ Departments of Physics and of Mathematics, University of British Columbia, \\ Vancouver, B. C., Canada
}

Received June 11, 1974; in revised form March 27, 1975

\begin{abstract}
The group $G$ of unitary elements of a maximal abelian von Neumann algebra on a separable, complex Hilbert space $H$ acts as a group of automorphisms on the CAR algebra $\mathscr{A}(H)$ over $H$. It is shown that the set of $G$-invariant states is a simplex, isomorphic to the set of regular probability measures on a $w^{*}$-compact set $S$ of $G$-invariant generalized free states. The GNS Hilbert space induced by an arbitrary $G$-invariant state on $\mathscr{A}(H)$ supports a *-representation of $C(S)$; the canonical map of $\mathscr{A}(H)$ into $C(S)$ can then be locally implemented by a normal, $G$-invariant conditional expectation.
\end{abstract}

In this paper we shall define observable Fermion number densities on the spectra of complete one particle observables and study the classical fields which they generate.

Let $\mathscr{A}(H)$ denote the $C^{*}$-algebra of the Canonical Anticommutation Relations (CAR) over a complex, separable Hilbert space $H$. $H$ will be fixed throughout and $\mathscr{A}(H)$ denoted by $\mathscr{A} . \mathscr{A}$ is generated algebraically by the range of an antilinear map $f \rightarrow a(f)$ of $H$ into $\mathscr{A}$ obeying the CAR:

$$
a(f) a(g)+a(g) a(f)=0 \quad a^{*}(f) a(g)+a(g) a^{*}(f)=(g, f) \quad \forall f, g \in H .
$$

Let $u$ be a unitary operator on $H$. Then the map $a(f) \rightarrow a(u f)$ extends uniquely

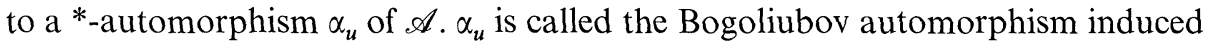
by $u$.

Let $\mathcal{O}$ be a self-adjoint operator on $\mathscr{H}$. $\mathcal{O}$ shall be called complete if its spectral family generates a maximal abelian von Neumann algebra $\mathscr{Y}$ on $H$. Let $(X, B, \mu)$ denote the spectral measure space of $\mathcal{O}$. By the well known isomorphism theorem (I $\S 7$ and III $\S 1$, Corollary 3 of Ref. [3]), completeness of $\mathcal{O}$ leads to identification of $\mathscr{H}$ with $\mathscr{L}^{2}(X, B, \mu)$ and of $\mathscr{Y}$ with $\mathscr{L}^{\infty}(X, B, \mu)$.

When $\mathcal{O}$ has discrete spectrum, the number density $N$ on $X$ is defined for each $x \in X$ by $N_{x}=a^{*}\left(\delta_{x}\right) a\left(\delta_{x}\right)$ where $\delta_{x}$ is the Kroenecker $\delta$-function at $x \in X$. The number density $N$ generates a classical field which is isomorphic to the lattice gas. One can also isolate the field and density by symmetry considerations (as we have remarked before [16]).

An observable in $\mathscr{A}$ is called $\mathcal{O}$-diagonal if it is diagonal in the Fock representation with respect to the basis formed by anti-symmetric products of eigenvectors 126.

* Supported by National Research Council of Canada grants NRC A 3119 NRC A 6570, NRC A 
of $\mathcal{O}$. The algebra of $\mathcal{O}$-diagonal observables is, at once, the classical field generated by the number density and the fixed point algebra for the unitary group $G(\mathscr{Y})$ of $\mathscr{Y}$ (which acts as Bogoliubov automorphisms): The $\mathcal{O}$-diagonal part of a correlation function is simply its average over $G(\mathscr{Y})$. Now, the $\mathcal{O}$-diagonal part of the one point functions $a^{*}(f) a(g)$ defines an operator-valued measure on $(X, B)$ which is continuous with respect to $\mu$, and whose Radon-Nikodym derivative is $N$. That is, if $D\left(a^{*}(f) a(g)\right)$ denotes the diagonal part of $a^{*}(f) a(g)$ then

$$
D\left(a^{*}(f) a(g)\right)=\int f(x) \bar{g}(x) N_{x} d \mu(x) \quad \forall f, g \in \mathscr{L}^{2}(X, B, \mu) .
$$

Thus, by focusing in the mean-values of correlation functions over $G(\mathscr{Y})$ one is naturally lead to the number density and its field. This is also the case when $\mathcal{O}$ does not have discrete spectrum.

Since the unitary group $G(\mathscr{Y})$ of an arbitrary maximal abelian von Neumann algebra $\mathscr{Y}$ may have poor ergodic properties even locally on $\mathscr{A}$, we shall, in effect, approximate $G(\mathscr{Y})$ by a net of compact subgroups, each of which has a unique invariant mean or conditional expectation on $\mathscr{A}$. In Section 2 , we associate to each partition of the spectrum of $\mathscr{Y}$ a conditional expectation on $\mathscr{A}$ which destroys the off-diagonal correlations between elements of the partition. Indexed by the partially ordered set of partitions, these conditional expectations form a net. In Section 3, we prove local convergence of the net in the representations induced by $G(\mathscr{Y})$-invariant states and isolate the local number densities and classical fields. In Section 4, we prove the set of $G(\mathscr{Y})$-invariant states to be a simplex and characterize its extreme points. Then, using the global consistency of the approximation scheme, we obtain the $C^{*}$-algebra of observables of the classical field over the spectrum of $\mathscr{Y}$.

The notation for von Neumann and $C^{*}$-algebras follows that of Refs. [4] and [5] with the following exceptions: If $\phi \in \mathscr{A}^{*}$ and $S \in \mathscr{A},\langle\phi ; S\rangle$ denotes the value of $\phi$ at $S$; if $f, g \in H$ (a Hilbert space), $\Lambda(f, g)$ denotes the vector form on $B(H)$ defined by $\langle\Lambda(f, g) ; S\rangle=(f, S g) \forall S \in B(H)$; the $\sigma$-topology of a von Neumann algebra is defined in Ref. [16]. The closure of a set $X$ in topology $\tau$ is denoted by $X^{-\tau}$, the closure of the linear span of $X$ by $[X]^{-\tau}$. The symmetric group of degree $N$ is denoted by $\mathscr{S}_{N}$ and $\operatorname{sgn}(p)$ denotes the signature of $p \in \mathscr{S}_{N}$.

\section{Conditional Expectations}

In this section we recall the definition of a conditional expectation on a $C^{*}$-algebra and reproduce, for completeness, two theorems giving sufficient conditions for its existence. These results are applied in $\S 2$ to the definition of a net of partial diagonal part operators.

0.1 . Definition [13]. Let $\mathscr{A}$ be a $C^{*}$-algebra with unit. A linear mapping $\mathscr{E}$ on $\mathscr{A}$ is said to be a conditional expectation if the following conditions are satisfied:

1. $\mathscr{E}(\mathbb{1})=\mathbb{1}$.

2. $S \geqq 0 \Rightarrow \mathscr{E}(S) \geqq 0$.

3. $\mathscr{E}(S \mathscr{E}(T))=\mathscr{E}(\mathscr{E}(S) T)=\mathscr{E}(S) \mathscr{E}(T) \quad \forall S, T \in \mathscr{A}$.

$\mathscr{E}$ is said to be faithful if $\mathscr{E}(S)=0, S \geqq 0 \Rightarrow S=0$.

The following theorem is a slight variant of the principle result of [16.1]. The proof is in the Appendix. 
0.2. Theorem. Let $\mathscr{A}$ be a $C^{*}$-algebra with unit, and let $G$ be an amenable discrete group of automorphisms of $\mathscr{A}$. Denote by $\mathscr{A}^{G}$ the $C^{*}$-algebra of all fixed points of $\mathscr{A}$ and by $\mathfrak{S}^{G}$ the set of all $G$-invariant states on $\mathscr{A}$. If there exists a $C^{*}$ subalgebra (with unit) $\mathscr{A}^{M}$ of $\mathscr{A}^{G}$ which separates $\mathfrak{S}^{G}$, then

1. If $\varrho$ is a state on $\mathscr{A}^{M}$, there exists a unique $G$-invariant state $\varrho$ on $\mathscr{A}$ extending $\hat{\varrho}$.

2. $\mathscr{A}^{M}=\mathscr{A}^{G}$.

3. If $S \in \mathscr{A}$, there exists $S^{G} \in \mathscr{A}$ such that $\left\{S^{G}\right\}=\mathscr{A}^{M} \cap \operatorname{Co}\{g S \mid g \in G\}^{-N}$.

4. The mapping $S \rightarrow S^{G}$ is a conditional expectation on $\mathscr{A}$ satisfying,

a) $S^{G}=S \quad \forall S \in \mathscr{A}^{G}$,

b) $(g S)^{G}=S^{G} \quad \forall g \in G ; \forall S \in \mathscr{A}$.

0.3. Definition [9]. Let $\mathscr{A}$ be a von Neumann algebra on a Hilbert space $H$ and let $G$ be a group of automorphisms of $\mathscr{A}$. Let $R^{+}(\mathscr{A}, G)$ denote the set of $G$-invariant, positive normal forms on $\mathscr{A}$. $\mathscr{A}$ is said to be $G$-finite if for every $S \in \mathscr{A}^{+}, S \neq 0$ there exists $\phi \in R^{+}(\mathscr{A}, G)$ such that $\langle\phi ; S\rangle \neq 0$.

Kovács and Szücs have obtained the following ergodic theorem.

0.4. Theorem. Let $\mathscr{A}$ be a von Neumann algebra and let $G$ be a group of automorphisms of $\mathscr{A}$. Let $\mathscr{A}^{G}$ denote the set of all fixed points of $\mathscr{A}$. Suppose that $\mathscr{A}$ is $G$-finite. Then, for every $T \in \mathscr{A}$, there exists $T^{G} \in \mathscr{A}$ such that $\left\{T^{G}\right\}=$ $\mathscr{A}^{G} \cap \operatorname{Co}\{g T \mid g \in G\}^{-\sigma}$. The mapping $T \rightarrow T^{G}$ is a normal, faithful conditional expectation on $\mathscr{A}$ such that

1. $(g T)^{G}=T^{G} \quad \forall g \in G ; \forall T \in \mathscr{A}$.

2. $T=T^{G} \quad \forall T \in \mathscr{A}^{G}$.

The mapping $T \rightarrow T^{G}$ is called the $G$-canonical map.

\section{The CAR}

Let $H$ be a separable, complex Hilbert space, and let $\mathscr{A}(H)$ be the $C^{*}$-algebra of the Canonical Anticommutation Relations (CAR) over $H$ [7]. As stated in the introduction, $\mathscr{A}(H)$ is generated by the range of an anti-linear mapping $a: H \rightarrow \mathscr{A}(H)$ satisfying the CAR: $a$ is continuous, with $\|a(f)\|=\|f\| \forall f \in H$; and $\mathscr{A}(H)$ is the closed linear span of the $n$-point correlation operators defined, for $N, M \in \mathbb{Z}^{+}$, $\left\{f_{n}\right\}_{n=1}^{N},\left\{g_{m}\right\}_{m=1}^{M} \subset H$ by

$$
A_{N M}\left(f_{1}, \ldots, f_{N} ; g_{1}, \ldots, g_{M}\right) \equiv a^{*}\left(f_{N}\right) \ldots a^{*}\left(f_{1}\right) a\left(g_{1}\right) \ldots a\left(g_{M}\right)
$$

By convention, $A_{00} \equiv 1, A_{10}(f) \equiv a^{*}(f), A_{01}(g) \equiv a(g)$.

Let $\subseteq$ be the set of all states on $\mathscr{A}(H)$. A state $\omega \in \mathbb{S}$ is determined by linearity and by continuity from its $n$-point correlation functions defined, for $N, M \in \mathbb{Z}^{+}$, $\left\{f_{n}\right\}_{n=1}^{N},\left\{g_{m}\right\}_{m=1}^{M} \subset H$, by

$$
W_{N M}\left(f_{1}, \ldots, f_{N} ; g_{1}, \ldots, g_{M}\right) \equiv\left\langle\omega ; A_{N M}\left(f_{1}, \ldots, f_{N} ; g_{1}, \ldots, g_{M}\right)\right\rangle \text {. }
$$

A state $\omega$ is said to be a gauge invariant generalized free state if its $n$-point correlation functions have the form;

$$
\begin{gathered}
W_{N M}\left(f_{1}, \ldots, f_{N} ; g_{1}, \ldots, g_{M}\right)=\delta_{M, N} \operatorname{det}\left\{W_{11}\left(f_{n}, g_{m}\right)\right\} \forall N, M \in \mathbb{Z}^{+} ; \\
\left\{f_{n}\right\}_{n=1}^{N},\left\{g_{m}\right\}_{m=1}^{M} \subset H .
\end{gathered}
$$


$F(H)$ denotes the set of all gauge invariant generalized free states on $\mathscr{A}$. If $\omega$ is a gauge invariant generalized free state there exists a unique operator $A$ on $H$ such $W_{11}(f ; g)=(g, A f) \forall f, g \in H$. It follows that $0 \leqq A \leqq 1$. Conversely, if $A$ is an operator on $H$ such that $0 \leqq A \leqq 1$, then there exists [2] a unique gauge invariant generalized free state $\omega_{A}$ such that

$$
\left\langle\omega_{A} ; a^{*}(f) a(g)\right\rangle=(g, A f) \quad \forall f, g \in H .
$$

Let $u$ be a unitary operator on $H$. Then, there exists a unique *-automorphism $\alpha_{u}$ of $\mathscr{A}(H)$ such that $\alpha_{u}(a(f))=a(u f) \forall f \in H$. The mapping $\alpha: U(H) \rightarrow \operatorname{Aut} \mathscr{A}(H)$ of the unitary group $U(H)$ of $H$ into the automorphisms group of $\mathscr{A}(H)$ is a strongly continuous homomorphism, when the former is equipped with its strong operator topology.

If $H^{\prime}$ is a closed subspace of $H$, we shall denote by $\mathscr{A}\left(H^{\prime}\right)$ the $C^{*}$-subalgebra of $\mathscr{A}(H)$ generated by

$$
\left\{A_{N M}\left(f_{1}, \ldots, f_{N} ; g_{1}, \ldots, g_{M}\right) \mid N, M \in \mathbb{Z}^{+} ;\left\{f_{n}\right\}_{n=1}^{N},\left\{g_{m}\right\}_{m=1}^{M} \subset H^{\prime}\right\}
$$

\section{A Net of Conditional Expectations}

Let $\mathscr{Y}$ be a maximal abelian von Neumann algebra on $H$, and let $G(\mathscr{Y})$ denote the unitary group of $\mathscr{Y}$.

2.0. Definition. Let $\tilde{E}=\left\{E_{\alpha}\right\}_{\alpha \in I}$ be a family of non-null, mutually orthogonal projectors in $\mathscr{Y}$ such that $\Sigma E_{\alpha}=\mathbb{1}_{H} . \tilde{E}$ is called a $\mathscr{Y}$-partition of $H$. Let $\Gamma(\mathscr{Y})$ denote the set of all $\mathscr{Y}$-partitions of $H$.

The set $\Gamma(\mathscr{Y})$ is partially ordered by refinement. That is, $\tilde{E}$ is said to refine $\tilde{F}$ if, for each $E \in \tilde{E}$ and $F \in \tilde{F}$, we have $E F=0$ or $E$. We write $\tilde{E} \geqq \tilde{F}$. One readily verifies that $(\Gamma(\mathscr{Y}), \geqq)$ is a partially ordered and directed set with $\tilde{E} \vee \tilde{F} \equiv\left\{E_{k} F_{j}\right\}$.

The directed set $(\Gamma(\mathscr{Y})$, $\geqq)$ will serve to index $a$ net of conditional expectations. The remainder of this section is aimed at defining a conditional expectation for each partition $\tilde{E} \in \Gamma(\mathscr{Y})$.

Let $\tilde{E}$ be a $\mathscr{Y}$-partition of $H$. We shall denote by $G(\tilde{E})$ the unitary group of the abelian von Neumann algebra $\mathscr{Y}(\tilde{E})$ generated by $\tilde{E} . G(\tilde{E})$ is represented in Aut $\mathscr{A}$ by $\alpha$ (see above). We denote the set of $G(\tilde{E})$-invariant elements of $\mathscr{A}$ (resp. $\Xi)$ by $\mathscr{A}^{G(\tilde{E})}$ (resp. $\left.\mathfrak{S}^{G(\tilde{E})}\right)$. If $\tilde{E}=\{\mathbb{1}\}$, then $G(\tilde{E})$ is the gauge group $\theta$.

2.1. Lemma. Let $\tilde{E}=\left\{E_{j}\right\}_{j \in J}$ be a $\mathscr{Y}$-partition of $H$; let, for each $j \in J, \mathscr{A}\left(E_{j} H\right)^{\theta}$ be the $C^{*}$-subalgebra of $\mathscr{A}$ generated by

$$
\left\{A_{N M}\left(f_{1}, \ldots, f_{N} ; g_{1}, \ldots, g_{M}\right) \mid N=M \in \mathbb{Z}^{+} ;\left\{f_{n}\right\}_{n=1}^{N},\left\{g_{m}\right\}_{m=1}^{M} \subset E_{j} H\right\} ;
$$

and, let $\bigotimes_{j} \mathscr{A}\left(E_{j} H\right)^{\theta}$ denote the $C^{*}$-subalgebra of $\mathscr{A}$ generated by $\bigcup_{j} \mathscr{A}\left(E_{j} H\right)^{\theta}$. Then, $\bigotimes_{j} \mathscr{A}\left(E_{j} H\right)^{\theta}$ separates $\mathfrak{S}^{G(\tilde{E})}$.

Proof. Let $\phi$ and $\phi^{1} \in \mathbb{S}^{G(E)}$ with $\phi \neq \phi^{1}$. There exists $N, M \in \mathbb{Z}^{+},\left\{f_{n}\right\}_{n=1}^{N}$, $\left\{g_{m}\right\}_{m=1}^{M} \subset H$ such that $\left\langle\phi-\phi^{1} ; A_{N M}\left(f_{1}, \ldots, f_{N} ; g_{1}, \ldots, g_{M}\right)\right\rangle \neq 0$. By linearity, and by continuity, there exists $\left\{j_{n}\right\}_{n=1}^{N},\left\{i_{m}\right\}_{m=1}^{M} \subset J$ such that

$$
\left\langle\phi-\phi^{1} ; A_{N M}\left(E_{j_{1}} f_{1}, \ldots, E_{j_{N}} f_{N} ; E_{i_{1}} g_{1}, \ldots, E_{i_{M}} g_{M}\right)\right\rangle \neq 0 \text {. }
$$


Let, for each $j \in J, N_{j} \equiv \operatorname{Card}\left\{n \mid j=j_{n}\right\}$ and $M_{j} \equiv \operatorname{Card}\left\{m \mid j=i_{m}\right\}$. Now, if $N_{j_{0}} \neq M_{j_{0}}$ for some $j_{0} \in J, G(\tilde{E})$-invariance of $\phi-\phi^{1}$ implies, with the choice $\mathbb{1}+\left[\exp \left(i \Pi /\left|N_{j_{0}}-M_{j_{0}}\right|\right)-1\right] E_{j_{0}} \in G(\tilde{E})$, that the LHS of preceding expression vanishes. Thus, $N_{j}=M_{j} \forall j \in J$. But this implies

$$
A_{N M}\left(E_{j_{1}} f_{1}, \ldots, E_{j_{N}} f_{N} ; E_{i_{1}} g_{1}, \ldots, E_{i_{M}} g_{M}\right) \subset \bigotimes_{j} \mathscr{A}\left(E_{j} H\right)^{\theta} .
$$

Since $G(\tilde{E})$ is amenable, and since $\bigotimes_{j} \mathscr{A}\left(E_{j} H\right)^{\theta} \cong \mathscr{A}^{G(\tilde{E})}$, Lemma 2.1 fulfills the remaining hypothesis of Theorem 0.2 . We have therefore the following:

2.2. Theorem. Let $\tilde{E}$ be a $\mathscr{Y}$-partition of $H$. There exists a unique $G(\tilde{E})$-invariant conditional expectation $\mathscr{E}(\mid \tilde{E})$ on $\mathscr{A}$ whose range is $\bigotimes \mathscr{A}\left(E_{j} H\right)^{\theta}=\mathscr{A}^{G(\tilde{E})}$.

2.3. Corollary. $\mathscr{E}\left(a^{*}(f) a(g) \mid \tilde{E}\right)=\Sigma_{j} a^{*}\left(E_{j} f\right) a\left(E_{j} g\right) \quad \forall f, g \in H$.

2.4. Corollary. Let $\tilde{E} \geqq \tilde{F}$ be two $\mathscr{Y}$-partitions of H. Then, $\mathscr{E}(\mathscr{E}(S \mid \tilde{F}) \mid \tilde{E})=\mathscr{E}(S \mid \tilde{E}) \quad \forall S \in \mathscr{A}$.

Proof. Since $G(\tilde{E}) \supseteqq G(\tilde{F})$ the result is immediate from Theorem 0.2 (3).

2.5. Corollary. Let $\tilde{E} \geqq \tilde{F}$ be two $\mathscr{Y}$-partitions of $H$. Then, $\mathscr{E}(\mathscr{A} \mid \tilde{E}) \cong \mathscr{E}(\mathscr{A} \mid \tilde{F})$.

We have therefore defined a net $\{\mathscr{E}(\mid \tilde{E})\}_{\tilde{E} \in \Gamma(\mathscr{\vartheta})}$ of conditional expectations on $\mathscr{A}$ with decreasing range (2.5) and the lattice property 2.4 .

We now turn to the implementation of this net on some representations of $\mathscr{A}$.

Let $\tilde{E}$ be a $\mathscr{Y}$-partition of $H$; let $\phi$ be a $G(\tilde{E})$-invariant state on $\mathscr{A}$; let $\left(\pi_{\phi}, U_{\phi}\right.$, $\left.H_{\phi}, \Phi\right)$ be the cyclic, covariant representation of $(\mathscr{A}, G(\tilde{E}))$ associated to $\mathscr{A}$ via the Gelfand-Naimark-Segal construction $[12,6.22]$. Since $\mathscr{A}$ is simple [15], it follows that $\pi_{\phi}$ is invertible. We can therefore define $\mathscr{E}_{\phi}(\mid \tilde{E}) \equiv \pi_{\phi} \mathscr{E}\left(\pi_{\phi}^{-1} \mid \tilde{E}\right): \pi_{\phi}(\mathscr{A}) \rightarrow$ $\pi_{\phi}\left(\mathscr{A}^{G(\tilde{E})}\right)$. For each $g \in G(\tilde{E})$ define the automorphism $\hat{\alpha}_{g}: \pi_{\phi}(\mathscr{A})^{\prime \prime} \rightarrow \pi_{\phi}(\mathscr{A})^{\prime \prime}$ by $\hat{\alpha}_{g} S=U_{\phi}(g) S U_{\phi}(g)^{*} \forall S \in \pi_{\phi}(\mathscr{A})^{\prime \prime}$. The mapping $\hat{\alpha}: G(\tilde{E}) \rightarrow \operatorname{Aut} \pi_{\phi}(\mathscr{A})^{\prime \prime}$ is a homomorphism such that $\hat{\alpha}_{g} \pi_{\phi}(S)=\pi_{\phi}\left(\alpha_{g} S\right) \forall S \in \mathscr{A}$. Accordingly, if we can prove that $\pi_{\phi}(\mathscr{A})^{\prime \prime}$ is $G(\tilde{E})$-finite $(0.3)$, the $G(\tilde{E})$-canonical map $(0.4)$ will be a normal, $G(\tilde{E})$ invariant conditional expectation on $\pi_{\phi}(\mathscr{A})^{\prime \prime}$ whose restriction to $\pi_{\phi}(\mathscr{A})$ is $\mathscr{E}_{\phi}(\mid \tilde{E})$.

By continuity, it will be the unique normal extension of $\mathscr{E}_{\phi}(\mid \tilde{E})$ to $\pi_{\phi}(\mathscr{A})^{\prime \prime}$. In that event we shall denote the $G(\tilde{E})$-canonical map by $\hat{\mathscr{E}}_{\phi}(\mid \tilde{E})$.

2.6. Lemma. Let $\tilde{E}=\left\{E_{j}\right\}_{j \in J}$ be a $\mathscr{Y}$-partition of $H$, and let $\phi$ be a $G(\tilde{E})$-invariant state. Then with the above notation, $\pi_{\phi}(\mathscr{A})^{\prime \prime}$ is $G(\tilde{E})$-finite.

Proof. It suffices to exhibit a complete orthonormal set of simultaneous eigenfunctions for $U_{\phi}(G(\tilde{E}))$. To this end, let $\mathscr{M}=\bigcup_{j}\left\{E_{j} f \mid f \in H\right\}$, and let

$$
\mathscr{M}_{\phi} \equiv \bigcup_{N, M}\left\{\pi_{\phi}\left(A_{N M}\left(f_{1}, \ldots, f_{N} ; g_{1}, \ldots, g_{M}\right)\right) \Phi \mid\left\{f_{n}\right\}_{n=1}^{N},\left\{g_{m}\right\}_{m=1}^{M} \subset \mathscr{M}\right\} .
$$

Clearly, the linear span of $\mathscr{M}_{\phi}$ is dense in $H_{\phi}$. Since $U_{\phi}(g) \Phi=\Phi \forall g \in G(\tilde{E})$, there exists, for $g \in G(\tilde{E})$ and $\Psi \in \mathscr{M}_{\phi}$, a unique $\theta(g, \Psi) \in[0,2 \Pi)$ such that $U_{\phi}(g) \Psi=$ $\exp [i \theta(g, \Psi)] \Psi$. Two vectors $\Psi$ and $\Psi^{1} \in \mathscr{M}_{\phi}$ are said to be equivalent $\Leftrightarrow$ if $\theta(g, \Psi)=$ $\theta\left(g, \Psi^{1}\right) \forall g \in G(\tilde{E}) . \mathscr{M}_{\phi}$ is thus divided into disjoint equivalence classes $\mathscr{M}_{\phi}^{\alpha}$. Let $\theta(g, \alpha) \equiv \theta(g, \Psi)$ for $\Psi \in \mathscr{M}_{\phi}^{\alpha}, g \in G(\tilde{E})$, and let $P_{\phi}^{\alpha}$ denote the projector upon 
the closed linear span $H_{\phi}^{\alpha}$ of $\mathscr{M}_{\phi}^{\alpha}$. One readily sees that $P_{\phi}^{\alpha} P_{\phi}^{\alpha^{\prime}}=0 \forall \alpha \neq \alpha^{\prime}, \Sigma P_{\phi}^{\alpha}=\mathbb{1}$, and

$$
U_{\phi}(g)=\Sigma_{\alpha} \exp (i \theta(g, \alpha)) P_{\phi}^{\alpha} \forall g \in G(\tilde{E}) .
$$

From Lemma 2.6 and the preceding discussion we have the following.

2.7. Theorem. Let $\phi$ be a $G(\mathscr{Y})$-invariant state on $\mathscr{A}$. Then, with the above notation and definitions, there exists a net $\left\{\hat{\mathscr{E}}_{\phi}(\mid \tilde{E})\right\}_{\tilde{E} \in \Gamma(\mathscr{Y})}$ of normal conditional expectations on $\pi_{\phi}(\mathscr{A})^{\prime \prime}$ such that

1. For each $\tilde{E} \in \Gamma(\mathscr{Y}), \hat{\mathscr{O}}_{\phi}(\mid \tilde{E})$ is a normal, $G(\tilde{E})$-invariant conditional expectation on $\pi_{\phi}(\mathscr{A})^{\prime \prime}$ whose range is $\left(\pi_{\phi}(\mathscr{A})^{\prime \prime}\right)^{G(\tilde{E})}$.

2. $\hat{\mathscr{E}}_{\phi}(\mid \tilde{E})$ is the unique normal extension of $\mathscr{E}_{\phi}(\mid \tilde{E})$.

3. If $\tilde{E} \geqq \tilde{F}$, then

$$
\hat{\mathscr{E}}_{\phi}\left(\hat{\mathscr{E}}_{\phi}(S \mid \tilde{F}) \mid \tilde{E}\right)=\hat{\mathscr{E}}_{\phi}(S \mid \tilde{E}) \forall S \in \pi_{\phi}(\mathscr{A})^{\prime \prime}
$$

4. If $\tilde{E} \geqq \tilde{F}$, then

$$
\hat{\mathscr{E}}_{\phi}\left(\pi_{\phi}(\mathscr{A})^{\prime \prime} \mid \tilde{E}\right) \cong \hat{\mathscr{E}}_{\phi}\left(\pi_{\phi}(\mathscr{A})^{\prime \prime} \mid \tilde{F}\right) \text {. }
$$

Remark. In the remainder of the paper we suppress the " ".

\section{A Local Theory}

In this section we investigate the convergence of the net $\left\{\mathscr{E}_{\phi}(\mid \tilde{E})\right\}_{\tilde{E} \in \Gamma(\mathscr{y})}$ of normal conditional expectations on $\pi_{\phi}(\mathscr{A})^{\prime \prime}$ defined in Theorem 2.7 for a maximal abelian von Neumann algebra $\mathscr{Y}$ on $H$ and $G(\mathscr{Y})$-invariant state on $\mathscr{A}$.

Due to the totally dissimilar behavior of the net for the atomic and nonatomic parts of $\mathscr{Y}$ we make the following:

3.0. Definition. Let $\mathscr{Y}$ be an abelian von Neumann algebra on $H$. A non-null projector $A \in \mathscr{Y}$ is said to be an atom of $\mathscr{Y}$ if for every projector $P$ of $\mathscr{Y}$ we have $A P=0$ or $A$. Denote the set of all atoms of $\mathscr{Y}$ by $A(\mathscr{Y})=\left\{A_{i}\right\}_{i \in I}$; let $Y_{A}=\vee A(\mathscr{Y})$ and $Y_{N}=1-Y_{A}$.

We remark that (1) since distinct atoms are mutually orthogonal, $Y_{A}=\Sigma A_{i}$, and (2) since $H$ is separable, Card $I \leqq \aleph_{0}$.

Let $\Omega \in H$ be a separating vector for the maximal abelian von Neumann algebra $\mathscr{Y}$ on $H[4,1 \S 7]$. For each element $F$ of $\mathscr{Y}$ we define the vector $f=F \Omega \in H$. The mapping $F \rightarrow f$ is an injection of $\mathscr{Y}$ onto a dense subset of $H$. Throughout the remainder of this section $\Omega$ will denote a fixed separating vector for $\mathscr{Y}$.

Let $P_{N}$ denote the set of all projectors $P$ of $\mathscr{Y}$ such that $P Y_{N}=P$. Clearly $P_{N}$ is a Boolean $\sigma$-algebra; the mapping $P \rightarrow \omega(P) \equiv(\Omega, P \Omega)$ is a finite positive measure on $P_{N}$; and $\left(P_{N}, \omega\right)$ is a finite, separable, non-atomic measure algebra. Therefore, $\left(P_{N}, \omega\right)$ is isomorphic to $\left(\left[0, \omega\left(Y_{N}\right)\right], d x\right)[8,41 \mathrm{C}]$.

3.1. Definition. Let $\tilde{E}^{0} \equiv\left\{Y_{N}\right\} \cup A(\mathscr{Y})$.

An increasing sequence $\left\{\tilde{E}^{n}\right\}_{n=1}^{\infty}$ of $\mathscr{Y}$-partitions of $H$ is said to be dense if 1) $\tilde{E}^{1} \geqq \tilde{E}^{0}$, and if 2 ) to every projector $F \in P_{N}$, and to every $\varepsilon>0$, there corresponds a positive integer $n_{0}$ and a projector $E$ which is a union of projectors of $\tilde{E}^{n_{0}}$ and is such that

$$
\omega(F(1-E)+E(1-F))<\varepsilon .
$$


Theorems $41 \mathrm{~B}$ and $41 \mathrm{C}$ of Ref. [8] assure the existence of an abundance of dense, increasing $\mathscr{Y}$-partitions of $H$.

\section{3.a A Local Convergence Theorem}

3.2. Theorem. Let $\mathscr{Y}$ be a maximal abelian von Neumann algebra on $H$; let $\phi$ be a $G(\mathscr{Y})$-invariant state on $\mathscr{A}$; let $\left\{\mathscr{E}_{\phi}(\mid \tilde{E})\right\}_{\tilde{E} \in \Gamma(\mathscr{Y})}$ be the net of conditional expectations on $\pi_{\phi}(\mathscr{A})^{\prime \prime}$ defined in Theorem 2.7. Then, there exists a unique normal conditional expectation $\mathscr{E}_{\phi}(\mid \mathscr{Y})$ on $\pi_{\phi}(\mathscr{A})^{\prime \prime}$ such that

$$
\sigma-\lim _{\Gamma(\mathscr{Y}) \mathscr{E}_{\phi}}\left(\pi_{\phi}(S) \mid \tilde{E}\right)=\mathscr{E}_{\phi}\left(\pi_{\phi}(S) \mid \mathscr{Y}\right) \quad \forall S \in \mathscr{A} .
$$

Further, 0) $\mathscr{E}_{\phi}(\mid \mathscr{Y})$ has abelian range.

$$
\text { 1) } \begin{aligned}
& \mathscr{E}_{\phi}\left(U_{\phi}(g) S U_{\phi}(g)^{*} \mid \mathscr{Y}\right)=\mathscr{E}_{\phi}(S \mid \mathscr{Y}) \quad \forall g \in G(\mathscr{Y}) . \\
& \text { 2) } U_{\phi}(g) \mathscr{E}_{\phi}(S \mid \mathscr{Y}) U_{\phi}(g)^{*}=\mathscr{E}_{\phi}(S \mid \mathscr{Y}) \quad \forall S \in \pi_{\phi}(\mathscr{A})^{\prime \prime} . \\
& \text { 3) } \mathscr{E}_{\phi}\left(\pi_{\phi}\left(A_{N M}\left(f_{1}, \ldots, f_{N} ; g_{1}, \ldots, g_{M}\right)\right) \mid \mathscr{Y}\right) \\
&=\delta_{M, N} \sum_{p \in \mathscr{S}_{N}} \operatorname{sgn}(p) \prod_{n=1}^{N} \mathscr{E}_{\phi}\left(\pi_{\phi}\left(A_{11}\left(f_{n} ; g_{p(n)}\right) \mid \mathscr{Y}\right)\right. \\
& \equiv \delta_{M, N} \operatorname{Det}\left\{\mathscr{E}_{\phi}\left(\pi_{\phi}\left(A_{11}\left(f_{n} ; g_{m}\right)\right) \mid \mathscr{Y}\right)\right\} \forall N, M \in \mathbb{Z}^{+} ; \\
& \quad \forall\left\{f_{n}\right\}_{n=1}^{N},\left\{g_{m}\right\}_{m=1}^{M} C H . \\
& \text { 4) } \mathscr{E}_{\phi}\left(\pi_{\phi}(\mathscr{A})^{\prime \prime} \mid \mathscr{Y}\right)=\left\{\mathscr{E}_{\phi}\left(\pi_{\phi}\left(A_{11}(f ; g)\right) \mid \mathscr{Y}\right) \mid f, g \in H\right\}^{\prime \prime} .
\end{aligned}
$$

The proof of this result is punctuated with several lemmas and propositions.

3.3. Remark. The proof of $\sigma$-convergence of the nets of operators $\left\{\mathscr{E}_{\phi}\left(\pi_{\phi}(S) \mid \tilde{E}\right)\right\}_{\tilde{E} \in \Gamma(\mathscr{Y})}$, which results in Proposition 3.11, employs the following artifice. Since, for each $S \in \mathscr{A}$ and $\mathscr{Y}$-partition $\tilde{E},\left\|\mathscr{E}_{\phi}\left(\pi_{\phi}(S) \mid \tilde{E}\right)\right\| \leqq\|S\|$ there exists, by $\sigma$-compactness of bounded spheres of $\pi_{\phi}(\mathscr{A})^{\prime \prime}$, a $\sigma$-convergent subnet: We chose one and denote its limit point by $\mathscr{E}_{\phi}^{0}\left(\pi_{\phi}(S) \mid \mathscr{Y}\right)$, finally proving that

$$
\mathscr{E}_{\phi}^{0}\left(\pi_{\phi}(S) \mid \mathscr{Y}\right)=\sigma-\lim _{\Gamma(\mathscr{Y})} \mathscr{E}_{\phi}\left(\pi_{\phi}(S) \mid \tilde{E}\right)
$$

This method of proof has the advantage that if $P$ is a projector on $H_{\phi}$, and if the net $\left\{P \mathscr{E}_{\phi}\left(\pi_{\phi}(S) \mid \tilde{E}\right) P\right\}_{\tilde{E} \in \Gamma(\mathscr{Y})}$ can be shown to be ultra-weakly convergent, then its limit point is $P \mathscr{E}_{\phi}^{0}\left(\pi_{\phi}(S) \mid \mathscr{Y}\right) P$. We remark further that since the weak-operator and ultra-weak operator topologies on $B\left(H_{\phi}\right)$ coincide on bounded spheres, weak operator convergence of the required nets is sufficient to ensure $\sigma$-convergence.

The following argument appears often enough to warrant abbreviation: If two vectors $\Psi, \Psi^{1} \in H_{\phi}$ are eigenvectors of a unitary operator $g$ with different eigenvalues, then they are orthogonal. We shall say " $\left(\Psi, \Psi^{1}\right)=0$ due to $g$-invariance", leaving the task of verifying that $\Psi$ and $\Psi^{1}$ belong to different eigensubspaces of $g$ to the reader.

Preparatory to proving Proposition 3.11, we make the following definitions and remarks. Let $D \in \mathscr{Y}$ be a projector; let $G(D)=\{\mathbb{1}+(g-1) D \mid g \in G(\mathscr{Y})\} \subseteq G(\mathscr{Y})$; and if $\phi$ is a $G(\mathscr{Y})$-invariant state on $\mathscr{A}$, let $H_{\phi}(D)=\left\{\Psi \in H_{\phi} \mid U_{\phi}(g) \Psi=\Psi \forall g \in G(D)\right\}$. 
Define the family of projectors $\left\{Q_{\phi}(D)_{i}\right\}_{i=1}^{4}$ as follows (recall that $d \equiv D \Omega$ where $\Omega$ is a fixed cyclic vector for $\mathscr{Y}$ in $H$ ).

$$
\begin{aligned}
& Q_{\phi}(D)_{1} H_{\phi} \equiv\left[\pi_{\phi}(a(d)) H_{\phi}(D)\right]^{-} \\
& Q_{\phi}(D)_{2} H_{\phi} \equiv\left[\pi_{\phi}\left(a^{*}(d)\right) H_{\phi}(D)\right]^{-} \\
& Q_{\phi}(D)_{3} H_{\phi} \equiv\left[\pi_{\phi}\left(a(d) a^{*}(d)\right) H_{\phi}(D)\right]^{-} \\
& Q_{\phi}(D)_{4} H_{\phi} \equiv\left[\pi_{\phi}\left(a^{*}(d) a(d)\right) H_{\phi}(D)\right]^{-} .
\end{aligned}
$$

From the CAR and $U_{\phi}(1-2 D)$-invariance it follows that these projectors are pairwise orthogonal. Let $Q_{\phi}(D) \equiv \Sigma Q_{\phi}(D)_{i}$.

If $D$ and $D^{\prime}$ be two orthogonal projectors of $\mathscr{Y}$, then $\left[Q_{\phi}(D), Q_{\phi}\left(D^{\prime}\right)\right]=0$. In fact, let $O_{\phi}(D)\left[\right.$ resp. $\left.O_{\phi}\left(D^{\prime}\right)\right]$ denote the projector upon $H_{\phi}(D)\left[\right.$ resp. $\left.H_{\phi}\left(D^{\prime}\right)\right]$. Since $O_{\phi}(D) \in U_{\phi}(G(D))^{\prime \prime}$ and $O_{\phi}\left(D^{\prime}\right) \in U_{\phi}\left(G\left(D^{\prime}\right)\right)^{\prime \prime}$ it follows that $\left[O_{\phi}(D), O_{\phi}\left(D^{\prime}\right)\right]=0$. From this, and the fact that $O_{\phi}\left(D^{\prime}\right) \in \pi_{\phi}(\mathscr{A}(\mathbb{C} d))^{\prime}, O_{\phi}\left(D^{\prime}\right) Q_{\phi}(D) H_{\phi} \subseteq Q_{\phi}(D) H_{\phi}$. Hence $\left[O_{\phi}\left(D^{\prime}\right), Q_{\phi}(D)\right]=0$ and $Q_{\phi}(D) O_{\phi}\left(D^{\prime}\right) H_{\phi} \subseteq O_{\phi}\left(D^{\prime}\right) H_{\phi}$. Consequently,

$$
Q_{\phi}(D) Q_{\phi}\left(D^{\prime}\right) H_{\phi} \subseteq Q_{\phi}\left(D^{\prime}\right) H_{\phi},
$$

proving the assertion.

3.4. Lemma. Let $D$ be a projector of $\mathscr{Y}$. Then, with the above definitions and notation,

strong $\lim _{\Gamma(\mathscr{Y})} Q_{\phi}(D) \mathscr{E}_{\phi}\left(\pi_{\phi}(N(d)) \mid \tilde{E}\right) Q_{\phi}(D)=Q_{\phi}(D) \mathscr{E}_{\phi}^{0}\left(\pi_{\phi}(N(d)) \mid \mathscr{Y}\right) Q_{\phi}(D)$

$\left(N(d) \equiv a^{*}(d) a(d)\right)$

Proof. We prove, for $i, i^{\prime}=1, \ldots, 4$, that each of the nets $(*)_{i i^{\prime}} \equiv$ $\left\{Q_{\phi}(D)_{i} \mathscr{E}_{\phi}\left(\pi_{\phi}(N(d)) \mid \tilde{E}\right) Q_{\phi}(D)_{i^{\prime}}\right\}_{\tilde{E} \in \Gamma(\mathscr{Y})}$ is strong-operator convergent. However, by $U_{\phi}((\mathbb{1}-D)+i D)$-invariance, the $\left(i, i^{\prime}\right)$-net vanishes for

$$
\begin{array}{ll}
i=1 ; i^{\prime}=2,3,4 & i=3 ; i^{\prime}=1,2 \\
i=2 ; i^{\prime}=1,3,4 & i=4 ; i^{\prime}=1,2 .
\end{array}
$$

Thus, the proof reduces to six cases:

Case 1. $i=i^{\prime}=1$. We prove the bounded net of positive operators to be increasing and therefore [4, Appendix 1] strong operator convergent. Let $\tilde{E} \geqq \tilde{F}$, and let $F_{k}=\Sigma_{j \in J(k)} E_{j}$, with the obvious notation. Let $\Psi=\pi_{\phi}(a(d)) \chi$ with $\chi \in H_{\phi}(D)$. Then

$$
\begin{aligned}
& \left(\Psi,\left\{\mathscr{E}_{\phi}\left(\pi_{\phi}(N(d)) \mid \tilde{F}\right)-\mathscr{E}_{\phi}\left(\pi_{\phi}(N(d)) \mid \tilde{E}\right)\right\} \Psi\right) \\
& =\Sigma_{k}\left(\chi, \pi_{\phi}\left[a^{*}(d)\left\{a^{*}\left(F_{k} d\right) a\left(F_{k} d\right)-\sum_{j \in J(k)} a^{*}\left(E_{j} d\right) a\left(E_{j} d\right)\right\} a(d)\right] \chi\right) \\
& =\Sigma_{k}\left(\chi, \pi_{\phi}\left[a^{*}\left(\left(\mathbb{1}-F_{k}\right) d\right)\{-\} a\left(\left(\mathbb{1}-F_{k}\right) d\right)\right] \chi\right) \\
& \quad+\Sigma_{k}\left(\chi, \pi_{\phi}\left[a^{*}\left(F_{k} d\right)\{-\} a\left(F_{k} d\right)\right] \chi\right) \\
& \quad+\Sigma_{k}\left(\chi, \pi_{\phi}\left[a^{*}\left(\left(\mathbb{1}-F_{k}\right) d\right)\{-\} a\left(F_{k} d\right)\right] \chi\right)+\text { c.c. }
\end{aligned}
$$


Now, (1) the first term on the RHS vanishes by $U_{\phi}\left(\mathbb{1}-2 D E_{j}\right)$-invariance for $j \in J(k)$ and all $k ;(2)$ the third and fourth terms vanish by $U_{\phi}\left(\mathbb{1}-2 D\left(\mathbb{1}-F_{k}\right)\right)$-invariance for all $k ;(3)$ since $a\left(F_{k} d\right)^{2}=0$, we are left with

$$
-\Sigma_{k}\left(\chi, \pi_{\phi}\left[a^{*}\left(F_{k} d\right)\left\{\sum_{j \in J(k)} a^{*}\left(E_{j} d\right) a\left(E_{j} d\right)\right\} a\left(F_{k} d\right)\right] \chi\right) \leqq 0 .
$$

Since $\Psi \in \pi_{\phi}(a(d)) H_{\phi}(D)$ was arbitrary, we have by continuity $\tilde{E} \geqq \tilde{F} \Rightarrow$ $Q_{\phi}(D)_{1} \mathscr{E}_{\phi}\left(\pi_{\phi}(N(d)) \mid \tilde{E}\right) Q_{\phi}(D)_{1} \geqq Q_{\phi}(D)_{1} \mathscr{E}_{\phi}\left(\pi_{\phi}(N(d)) \mid \tilde{F}\right) Q_{\phi}(D)_{1}$.

Case 2. $i=i^{\prime}=2$. We prove the net $(*)_{22}$ of positive operators to be decreasing and therefore strong-operator convergent. Let $\tilde{E} \geqq \tilde{F}$ and let $F_{k}=\sum_{j \in J(k)} E_{j}$. Let $\Psi=\pi_{\phi}\left(a^{*}(d)\right) \chi$ with $\chi \in H_{\phi}(D)$. Then, by an argument similar to that of Case 1 , we have;

$$
\begin{aligned}
& \left(\Psi,\left[\mathscr{E}_{\phi}\left(\pi_{\phi}(N(d)) \mid \tilde{F}\right)-\mathscr{E}_{\phi}\left(\pi_{\phi}(N(d)) \mid \tilde{E}\right)\right] \Psi\right) \\
& =\sum_{k}\left(\chi, \pi_{\phi}\left[a\left(F_{k} d\right)\left\{\left\|F_{k} d\right\|^{2}-\Sigma_{j \in J(k)} a^{*}\left(E_{j} d\right) a\left(E_{j} d\right)\right\} a^{*}\left(F_{k} d\right)\right] \chi\right) \geqq 0
\end{aligned}
$$

since

$$
\sum_{j \in J(k)}\left\|a^{*}\left(E_{j} d\right) a\left(E_{j} d\right)\right\| \leqq \sum_{j \in J(k)}\left\|E_{j} d\right\|^{2}=\left\|F_{k} d\right\|^{2} .
$$

Since $\Psi \in \pi_{\phi}\left(a^{*}(d)\right) H_{\phi}(D)$ was arbitrary, we have, by continuity,

$$
\tilde{E} \geqq \tilde{F} \Rightarrow Q_{\phi}(D)_{2} \mathscr{E}_{\phi}\left(\pi_{\phi}(N(d)) \mid \tilde{E}\right) Q_{\phi}(D)_{2} \leqq Q_{\phi}(D)_{2} \mathscr{E}_{\phi}\left(\pi_{\phi}(N(d)) \mid \tilde{F}\right) Q_{\phi}(D)_{2} .
$$

Case 3. $i=i^{\prime}=3$. We have (CAR),

$$
a(d) a^{*}(d) \mathscr{E}(N(d) \mid \tilde{E}) a(d) a^{*}(d)=\|d\|^{2} a(d) \mathscr{E}(N(d) \mid \tilde{E}) a^{*}(d)
$$$$
-a(d) a^{*}(d) \Sigma_{j}\left\|E_{j} d\right\|^{4} \text {. }
$$

Therefore,

$$
\begin{aligned}
& Q_{\phi}(D)_{3} \mathscr{E}_{\phi}\left(\pi_{\phi}(N(d)) \mid \tilde{E}\right) Q_{\phi}(D)_{3} \\
& =\|d\|^{-2} Q_{\phi}(D)_{3} \pi_{\phi}(a(d)) \mathscr{E}_{\phi}\left(\pi_{\phi}(N(d)) \mid \tilde{E}\right) \pi_{\phi}\left(a^{*}(d)\right) Q_{\phi}(D)_{3} \\
& \quad-\|d\|^{-4} Q_{\phi}(D)_{3} \pi_{\phi}\left(a(d) a^{*}(d)\right) Q_{\phi}(D)_{3}\left[\Sigma_{j}\left\|E_{j} d\right\|^{4}\right] .
\end{aligned}
$$

Now, (1) since $\pi_{\phi}\left(a^{*}(d)\right) Q_{\phi}(D)_{3}=Q_{\phi}(D)_{2} \pi_{\phi}\left(a^{*}(d)\right)$, strong-operator convergence of the net of first term on the RHS follows from Case 2; and since the net in $\mathbb{C}$, $\left\{\Sigma_{j}\left\|E_{j} d\right\|^{4}\right\}_{\tilde{E} \in \Gamma(\mathscr{Y})}$ is positive and decreasing, the net of second terms is norm and, a fortiori, strong-operator convergent.

Case 4. $i=i^{\prime}=4$. By an argument similar to that of Case 3, we have

$$
\begin{aligned}
& Q_{\phi}(D)_{4} \mathscr{E}_{\phi}\left(\pi_{\phi}(N(d)) \mid \tilde{E}\right) Q_{\phi}(D)_{4} \\
& =\|d\|^{-4} Q_{\phi}(D)_{4} \pi_{\phi}(N(d)) Q_{\phi}(D)_{4}\left(\Sigma_{j}\left\|E_{j} d\right\|^{4}\right) \\
& \quad+\|d\|^{-2} Q_{\phi}(D)_{4} \pi_{\phi}\left(a^{*}(d)\right) \mathscr{E}_{\phi}\left(\pi_{\phi}(N(d)) \mid \tilde{E}\right) \pi_{\phi}(a(d)) Q_{\phi}(D)_{4} .
\end{aligned}
$$

Now, since $\pi_{\phi}(a(d)) Q_{\phi}(D)_{4}=Q_{\phi}(D)_{1} \pi_{\phi}(a(d))$, strong-operator convergence of the net of second terms on the RHS follows from Case 1; (2) Convergence of the net of first terms follows as in Case 3. 
Case 5. $i=3, i^{\prime}=4$. We have (CAR)

$$
\begin{aligned}
& Q_{\phi}(D)_{3} \mathscr{E}_{\phi}\left(\pi_{\phi}(N(d)) \mid \tilde{E}\right) Q_{\phi}(D)_{4} \\
& =\|d\|^{-2} Q_{\phi}(D)_{3} \pi_{\phi}\left[a^{*}\left(\Sigma\left\|E_{j} d\right\|^{2} E_{j} d\right) a(d)\right] Q_{\phi}(D)_{4} .
\end{aligned}
$$

Now let, for each $\mathscr{Y}$-partition $\tilde{E}$ of $H$,

$$
f(d, \tilde{E})=\Sigma_{j}\left\|E_{j} d\right\|^{2} E_{j} d \text {. Let } f(d, \mathscr{Y})=\Sigma\left\|A_{i} d\right\|^{2} A_{i} d .
$$

Let, for each $M \in \mathbb{Z}^{+}, \tilde{E}^{M}$ be a $\mathscr{Y}$-partition of $H$ such that 1) $\tilde{E}^{M} \geqq \tilde{E}^{0} \equiv A(\mathscr{Y}) \cup\left\{Y_{N}\right\}$, and 2) $\left(\Omega, D Y_{N} E_{j}^{M} \Omega\right)=0$ or $\left(\Omega, D Y_{N} \Omega\right) / M \forall j$. Let $\tilde{F} \geqq \tilde{E}^{M}$ for fixed, but arbitrary, $M: E_{j}^{M}=\Sigma_{k \in K(j)} F_{k}$.

We have, $\|f(d, \mathscr{Y})-f(d, \tilde{F})\|^{2}=\Sigma_{k}\left\|F_{k} Y_{N} d\right\|^{4}=\Sigma_{j} \Sigma_{K(j)}\left\|F_{k} Y_{N} d\right\|^{4} \leqq \Sigma_{j}\left\|E_{j}^{M} Y_{N} d\right\|^{4}=$ $\left(\Omega, D Y_{N} \Omega\right)^{2} / M$. Thus $\lim _{\Gamma(\mathscr{Y})} f(d, \tilde{E})=f(d, \mathscr{Y})$, and consequently

$$
\begin{aligned}
& \lim _{\Gamma(\mathscr{Y})} Q_{\phi}(D)_{3} \mathscr{E}_{\phi}\left(\pi_{\phi}(N(d)) \mid \tilde{E}\right) Q_{\phi}(D)_{4} \\
& =\|d\|^{-2} Q_{\phi}(D)_{3} \pi_{\phi}\left[a^{*}(f(d, \mathscr{Y})) a(d)\right] Q_{\phi}(D)_{4} .
\end{aligned}
$$

Case 6. $i=4, i^{\prime}=3$. Case 6 follows from Case 5 by virtue of norm convergence.

3.5. Lemma. Let $\tilde{D}$ be a $\mathscr{Y}$-partition of $H$; let $\left\{F_{n}\right\}_{n=1}^{N}$ be a finite set of mutually orthogonal projectors of $\mathscr{Y}(\tilde{D})$ (i.e. $\left.F_{n}=\Sigma_{j \in J(n)} D_{j}\right)$; and let $P_{\phi}(\tilde{D}) H_{\phi} \equiv$ $\left[\pi_{\phi}\left(\mathscr{A}\left([\tilde{D} \Omega]^{-}\right)\right) \Phi\right]^{-}$.

Then

$\mathrm{S}-\lim _{\Gamma(\mathscr{Y})^{N}} P_{\phi}(\tilde{D}) \prod_{n=1}^{N} \mathscr{E}_{\phi}\left(\pi_{\phi}\left(N\left(f_{n}\right)\right) \mid \tilde{E}^{n}\right) P_{\phi}(\tilde{D})$

$$
=P_{\phi}(\tilde{D}) \prod_{n=1}^{N} \mathscr{E}_{\phi}^{0}\left(\pi_{\phi}\left(N\left(f_{n}\right)\right) \mid \mathscr{Y}\right) P_{\phi}(\tilde{D}) \text {. }
$$

Proof. Let $Q_{\phi}(n) \equiv \prod_{j \in J(n)} Q_{\phi}\left(D_{j}\right)$. It follows from Lemma 3.4 that, for each finite subset $J_{0}(n) \cong J(n)$,

$$
\begin{aligned}
& \mathrm{S}-\lim _{\Gamma(\mathscr{y})} Q_{\phi}(n) \mathscr{E}_{\phi}\left(\pi_{\phi}\left(N\left(\sum_{j \in J_{0}(n)} D_{j} f_{n}\right)\right) \mid \tilde{E}\right) Q_{\phi}(n) \\
& =Q_{\phi}(n) \mathscr{E}_{\phi}^{0}\left(\pi_{\phi}\left(N\left(\sum_{j \in J_{0}(n)} D_{j} f_{n}\right)\right) \mid \mathscr{Y}\right) Q_{\phi}(n) .
\end{aligned}
$$

Since, for $\tilde{E} \geqq \tilde{D}$, we have

$$
\begin{aligned}
& \left\|Q_{\phi}(n)\left\{\mathscr{E}_{\phi}\left(\pi_{\phi}\left(N\left(f_{n}\right)\right) \mid \tilde{E}\right)-\mathscr{E}_{\phi}\left(\pi_{\phi}\left(N\left(\sum_{j \in J_{0}(n)} D_{j} f_{n}\right)\right) \mid \tilde{E}\right)\right\} Q_{\phi}(n)\right\| \\
& \leqq\left\|\left(\mathbb{1}-\sum_{j \in J_{0}(n)} D_{j}\right) f_{n}\right\|^{2}
\end{aligned}
$$

it follows that

$$
\begin{aligned}
& \left\|Q_{\phi}(n)\left\{\mathscr{E}_{\phi}^{O}\left(\pi_{\phi}\left(N\left(f_{n}\right)\right) \mid \mathscr{Y}\right)-\mathscr{E}_{\phi}^{0}\left(\pi_{\phi}\left(N\left(\sum_{j \in J_{0}(n)} D_{j} f_{n}\right)\right) \mid \mathscr{Y}\right)\right\} Q_{\phi}(n)\right\| \\
& \leqq\left\|\left(\mathbb{1}-\sum_{j \in J_{0}(n)} D_{j}\right) f_{n}\right\|^{2}
\end{aligned}
$$

It then follows, by the triangle and Schwartz inequalities, from the above remarks that

$\mathrm{S}-\lim _{\Gamma(\mathscr{Y})} Q_{\phi}(n) \mathscr{E}_{\phi}\left(\pi_{\phi}\left(N\left(f_{n}\right)\right) \mid \tilde{E}\right) Q_{\phi}(n)=Q_{\phi}(n) \mathscr{E}_{\phi}^{0}\left(\pi_{\phi}\left(N\left(f_{n}\right)\right) \mid \mathscr{Y}\right) Q_{\phi}(n)$. 
By joint strong-operator continuity of products on bounded sets, and by virtue of the fact that, for $n \neq n^{\prime}$,

$$
\begin{aligned}
0=\left[Q_{\phi}(n), Q_{\phi}\left(n^{\prime}\right)\right] & =\left[\mathscr{E}_{\phi}\left(\pi_{\phi}\left(N\left(f_{n}\right)\right) \mid \tilde{E}\right), Q_{\phi}\left(n^{\prime}\right)\right] \\
& =\left[\mathscr{E}_{\phi}\left(\pi_{\phi}\left(N\left(f_{n}\right)\right) \mid \tilde{E}\right), \mathscr{E}_{\phi}\left(\pi_{\phi}\left(N\left(f_{n^{\prime}}\right)\right) \mid \tilde{F}\right)\right],
\end{aligned}
$$

we have

$$
\begin{aligned}
& \mathrm{S}-\lim _{\Gamma(\mathscr{g})^{N}} \prod_{n=1}^{N} Q_{\phi}(n) \prod_{n=1}^{N} \mathscr{E}_{\phi}\left(\pi_{\phi}\left(N\left(f_{n}\right)\right) \mid \tilde{E}^{n}\right) \prod_{n=1}^{N} Q_{\phi}(n) \\
& =\prod_{n=1}^{N} Q_{\phi}(n) \prod_{n=1}^{N} \mathscr{E}_{\phi}^{0}\left(\pi_{\phi}\left(N\left(f_{n}\right)\right) \mid \mathscr{Y}\right) \prod_{n=1}^{N} Q_{\phi}(n) .
\end{aligned}
$$

However, since $P_{\phi}(\tilde{D}) \leqq Q_{\phi}\left(D_{j}\right) \forall j$; it follows that $\prod_{n=1}^{N} Q_{\phi}(n) \geqq P_{\phi}(\tilde{D})$ : This, and the preceding statement, proves the lemma.

3.6. Lemma. Let $\left\{F_{n}\right\}_{n=1}^{N}$ be a finite family of mutually orthogonal projectors of $\mathscr{Y}$. Then,

$$
\mathrm{S}-\lim _{\Gamma(\mathscr{Y})^{N}} \prod_{n=1}^{N} \mathscr{E}_{\phi}\left(\pi_{\phi}\left(N\left(f_{n}\right)\right) \mid \tilde{E}^{n}\right)=\prod_{n=1}^{N} \mathscr{E}_{\phi}^{0}\left(\pi_{\phi}\left(N\left(f_{n}\right)\right) \mid \mathscr{Y}\right) .
$$

Proof. Let $\Gamma\left(\mathscr{Y} \mid\left\{F_{n}\right\}\right) \equiv\left\{\tilde{D} \in \Gamma(\mathscr{Y}) \mid F_{n} \in \tilde{D}^{\prime \prime} \forall n\right\}$. Clearly, $\Gamma\left(\mathscr{Y} \mid\left\{F_{n}\right\}\right)$ is a directed set and cofinal with $\Gamma(\mathscr{Y})$ : thus, since $[13,1.3 .1$ and Remark, p. 46] the linear span of the projectors of $\mathscr{Y}$ is norm-dense in $\mathscr{Y}$, since $\Omega$ is cyclic for $\mathscr{Y}$ in $H$, since $\Phi$ is cyclic for $\pi_{\phi}(\mathscr{A})$ in $H_{\phi}$ and since the $n$-point correlation operators $\left\{A_{N M}\right\}$ are continuous and multilinear, it follows that

$$
\mathrm{S}-\lim _{\Gamma\left(\mathscr{Y} \mid\left\{F_{n}\right\}\right)} P_{\phi}(\tilde{D})=\mathbb{1} .
$$

The result then follows from Lemma 3.5 via the inequality, valid for

$$
\begin{aligned}
& \tilde{D} \in \Gamma\left(\mathscr{Y} \mid\left\{F_{n}\right\}\right), \\
& \left|\left(\Psi,\left[\prod_{n=1}^{N} \mathscr{E}_{\phi}\left(\pi_{\phi}\left(N\left(f_{n}\right)\right) \mid \tilde{E}^{n}\right)-\prod_{n=1}^{N} \mathscr{E}_{\phi}^{0}\left(\pi_{\phi}\left(N\left(f_{n}\right)\right) \mid \mathscr{Y}\right)\right] \Psi\right)\right| \\
& \leqq\left|\left(P_{\phi}(\tilde{D}) \Psi,[-] P_{\phi}(\tilde{D}) \Psi\right)\right|+2 \prod_{n=1}^{N}\left\|f_{n}\right\|^{2}\left\|\left(\mathbb{1}-P_{\phi}(\tilde{D})\right) \Psi\right\| \\
& \cdot\left(2\|\Psi\|+\left\|\left(\mathbb{1}-P_{\phi}(\tilde{D})\right) \Psi\right\|\right) . \quad \square
\end{aligned}
$$

3.7. Lemma. Let $\left\{S_{n}\right\}$ be a Cauchy Sequence in $\mathscr{A}$ such that

$$
\mathrm{W}-\lim _{\Gamma(\mathscr{Y})} \mathscr{E}_{\phi}\left(\pi_{\phi}\left(S_{n}\right) \mid \tilde{E}\right)=\mathscr{E}_{\phi}^{\circ}\left(\pi_{\phi}\left(S_{n}\right) \mid \mathscr{Y}\right)
$$

for each $n \in \mathbb{Z}^{+}$. Then, if $S=\mathrm{n}_{n \rightarrow \infty} \lim _{n} S_{n}$

$$
\mathrm{W}-\lim _{\Gamma(\mathscr{Y})} \mathscr{E}_{\phi}\left(\pi_{\phi}(S) \mid \tilde{E}\right)=\mathscr{E}_{\phi}^{0}\left(\pi_{\phi}(S) \mid \mathscr{Y}\right)=\mathrm{n}_{n \rightarrow \infty} \lim _{\phi} \mathscr{E}_{\phi}^{0}\left(\pi_{\phi}\left(S_{n}\right) \mid \mathscr{Y}\right) .
$$

The proof is immediate from linearity and uniform boundedness (i.e. $\left.\left\|\mathscr{E}_{\phi}\left(\pi_{\phi}(S) \mid \tilde{E}\right)\right\| \leqq\|S\| \forall \tilde{E} \in \Gamma(\mathscr{Y})\right)$ of the maps $\left.\left\{\mathscr{E}_{\phi}(\mid \tilde{E})\right\}_{\tilde{E} \in \Gamma(\mathscr{Y})}\right)$.

3.8. Lemma. Let $N, M \in \mathbb{Z}^{+}$, and let $\left\{f_{n}\right\}_{n=1}^{N},\left\{g_{m}\right\}_{m=1}^{M} \subset H$. Then,

$$
\begin{aligned}
& \mathrm{w}-\lim _{\Gamma(\mathscr{Y}) \mathscr{E}_{\phi}}\left(\pi_{\phi}\left(A_{N M}\left(f_{1}, \ldots, f_{N} ; g_{1}, \ldots, g_{M}\right)\right) \mid \tilde{E}\right) \\
& =\mathscr{E}_{\phi}^{0}\left(\pi_{\phi}\left(A_{N M}\left(f_{1}, \ldots, f_{N} ; g_{1}, \ldots, g_{M}\right)\right) \mid \mathscr{Y}\right) \\
& =\delta_{M N} \sum_{p \in \mathscr{S}_{N}} \operatorname{sgn}(p) \prod_{n=1}^{N} \mathscr{E}_{\phi}^{0}\left(\pi_{\phi}\left(A_{11}\left(f_{n} ; g_{p(n)}\right)\right) \mid \mathscr{Y}\right) .
\end{aligned}
$$

As implied by the notation, the order of the product is unimportant. 
Proof. Let $\tilde{D}=\left\{D_{k}\right\}$ be a $\mathscr{Y}$-partition of $H$. Let, for each $1 \leqq n \leqq N$ (resp. $1 \leqq m \leqq M), D_{k_{n}}$ (resp. $D_{\kappa_{m}}$ ) be chosen from $\tilde{D}$; let $f_{n}=d_{k_{n}} \forall 1 \leqq n \leqq N$ (resp. $\left.g_{m}=d_{\kappa_{m}} \forall 1 \leqq m \leqq M\right)$. By the familiar invariance argument (e.g. Proof of 2.1) we have $\mathscr{E}_{\phi}\left(\pi_{\phi}\left(A_{N M}\left(f_{1}, \ldots, f_{N} ; g_{1}, \ldots, g_{M}\right)\right) \mid \tilde{D}\right)$

$$
\begin{aligned}
& =\left[\begin{array}{l}
0 \quad \text { if }(1) k_{n}=k_{n^{\prime}} \text { for } n \neq n^{\prime} \\
\quad \text { or }(2) \kappa_{m}=\kappa_{m^{\prime}} \text { for } m \neq m^{\prime} \\
\quad \begin{array}{l}
\operatorname{or}(3)\left\{k_{n}\right\}_{n=1}^{N} \neq\left\{\kappa_{m}\right\}_{m=1}^{M} \\
\operatorname{sgn}(p) \prod_{n=1}^{N} E_{\phi}\left(\pi_{\phi}\left(A_{11}\left(d_{k_{n}} ; d_{k_{n}}\right)\right) \mid \tilde{D}\right), \text { where }
\end{array} \\
p \text { is the unique permutation such that } k_{n}=\kappa_{p(n)} \forall n
\end{array}\right. \\
& =\delta_{M, N} \sum_{p \in \mathscr{S}_{N}} \operatorname{sgn}(p) \prod_{n=1}^{N} \mathscr{E}_{\phi}\left(\pi_{\phi}\left(A_{11}\left(f_{n} ; g_{p(n)}\right)\right) \mid \tilde{D}\right) .
\end{aligned}
$$

Taking into account the orthogality of the $\left\{D_{k}\right\}$, one has, by operating on both sides by $\mathscr{E}_{\phi}(\mid \tilde{E})$, the above equality for $\tilde{D}$ replaced by $\tilde{E} \geqq \tilde{D}$. The result then follows for this special case from Lemma 3.6. Clearly, the order of the product is irrelevant. Since the linear span of the set of all projectors of $\mathscr{Y}$ is norm dense in $\mathscr{Y}[13,1.3 .1]$, and since $\Omega$ is cyclic for $\mathscr{Y}$ in $H$, the general case follows from linearity and continuity (3.7).

3.9. Definition. Let $D_{\phi}(\mathscr{Y})\left[\operatorname{resp} . D_{\phi}\left(\mathscr{Y}_{A}\right), D_{\phi}\left(\mathscr{Y}_{N}\right)\right]$ denote the $C^{*}$-algebra on $H_{\phi}$ generated by

$$
\left\{\mathscr{E}_{\phi}^{0}\left(\pi_{\phi}\left(a^{*}(f) a(g)\right) \mid \mathscr{Y}\right) \mid f, g \in H\left(\text { resp. } Y_{A} H, Y_{N} H\right)\right\} \cup\{\mathbb{1}\} .
$$

We remark that $(2.3) D_{\phi}(\mathscr{Y})$ is generated by $D_{\phi}\left(\mathscr{Y}_{A}\right) \cup D_{\phi}\left(\mathscr{Y}_{N}\right)$.

3.10. Lemma. Let $\mathscr{Z}_{\phi}=\pi_{\phi}(\mathscr{A})^{\prime \prime} \cap \pi_{\phi}(\mathscr{A})^{\prime}$. Then $D_{\phi}\left(\mathscr{Y}_{N}\right) \cong \mathscr{Z}_{\phi}$ and $D_{\phi}(\mathscr{Y})$ is abelian.

Proof. Since, by Lemma 3.8, $D_{\phi}\left(\mathscr{Y}_{N}\right) \cong \pi_{\phi}(\mathscr{A})^{\prime \prime}$, it remains to prove that $D_{\phi}\left(\mathscr{Y}_{N}\right) \subseteq \pi_{\phi}(\mathscr{A})^{\prime}$. It suffices to prove that $\left[\pi_{\phi}(a(f)), \mathscr{E}_{\phi}^{0}\left(\pi_{\phi}\left(a^{*}(g) a(l)\right) \mid \mathscr{Y}\right)\right]=$ $0 \forall f \in H ; \forall g, l \in Y_{N} H$. Since $\Omega$ is clearly cyclic for $Y_{N} \mathscr{Y}$ in $Y_{N} H$ it suffices by linearity and continuity to prove that, if $F, G, K$ be projectors of $\mathscr{Y}$, then $\left[\pi_{\phi}(a(f)), \mathscr{E}_{\phi}^{0}\left(\pi_{\phi}\left(A_{11}\left(Y_{N} g ; Y_{N} k\right)\right) \mid \mathscr{Y}\right)=0 ;\right.$ or,

$$
\mathrm{w}-\lim _{\Gamma(\mathscr{g})}\left[\pi_{\phi}(a(f)), \mathscr{E}_{\phi}\left(\pi_{\phi}\left(A_{11}\left(Y_{N} g ; Y_{N} k\right)\right) \mid \tilde{E}\right)\right]=0 \text {. }
$$

In fact, let $\tilde{F} \geqq \tilde{E}^{N}$ with the notation of Lemma 3.4, Case 5 but with $D=Y_{N}$. It then follows, by an elementary calculation, that $\|\left[\pi_{\phi}(a(f)), \mathscr{E}_{\phi}\left(\pi_{\phi}\left(A_{11}\left(Y_{N} g\right.\right.\right.\right.$; $\left.\left.\left.\left.Y_{N} k\right)\right) \mid \tilde{F}\right)\right] \|^{2} \leqq\left(\Omega, Y_{N} \Omega\right)^{3} / N$. This proves the first assertion. To prove that $D_{\phi}(\mathscr{Y})$ is abelian, it remains only to show that $D_{\phi}\left(\mathscr{Y}_{A}\right)$ is abelian. But, since $\mathscr{E}_{\phi}^{0}\left(\pi_{\phi}\left(A_{11}\left(Y_{A} f\right.\right.\right.$; $\left.\left.\left.Y_{A} g\right)\right) \mid \mathscr{Y}\right)=\Sigma \pi_{\phi}\left(a^{*}\left(A_{i} f\right) a\left(A_{i} g\right)\right)$, it follows that $D_{\phi}\left(\mathscr{Y}_{A}\right)$ is generated by $\left\{\pi_{\phi}\left(N\left(a_{i}\right)\right)\right\} \cup$ $\{\mathbb{1}\}$ and these clearly commute.

We now put together the results obtained so far.

3.11. Proposition. There exists a unique linear, continuous mapping $\mathscr{E}_{\phi}^{0}(\mid \mathscr{Y})$ : $\pi_{\phi}(\mathscr{A}) \rightarrow \pi_{\phi}(\mathscr{A})^{\prime \prime}$ such that

$$
\mathscr{E}_{\phi}^{0}\left(\pi_{\phi}(S) \mid \mathscr{Y}\right)=\sigma-\lim _{\Gamma(\mathscr{Y})} \mathscr{E}_{\phi}\left(\pi_{\phi}(S) \mid \tilde{E}\right) \quad \forall S \in \mathscr{A} .
$$


Further,

0) $\mathscr{E}_{\phi}^{0}(\mathbb{1} \mid \mathscr{Y})=\mathbb{1}$.

1) $\left\|\mathscr{E}_{\phi}^{0}(\mid \mathscr{Y})\right\|=1$.

2) $S \geqq 0 \Rightarrow \mathscr{E}_{\phi}^{0}\left(\pi_{\phi}(S) \mid \mathscr{Y}\right) \geqq 0$.

3) $\mathscr{E}_{\phi}^{0}\left(\pi_{\phi}\left(S^{*}\right) \mid \mathscr{Y}\right)=\mathscr{E}_{\phi}^{0}\left(\pi_{\phi}(S) \mid \mathscr{Y}\right)^{*} \quad \forall S \in \mathscr{A}$.

4) $\mathscr{E}_{\phi}^{0}\left(\mathscr{U}_{\phi}(g) \pi_{\phi}(S) \mathscr{U}_{\phi}(g) * \mid \mathscr{Y}\right)=\mathscr{E}_{\phi}^{0}\left(\pi_{\phi}(S) \mid \mathscr{Y}\right)$

$=\mathscr{U}_{\phi}(g) \mathscr{E}_{\phi}^{0}\left(\pi_{\phi}(S) \mid \mathscr{Y}\right) \mathscr{U}_{\phi}(g)^{*} \quad \forall S \in \mathscr{A} ; \forall g \in G(\mathscr{Y})$.

5) For $N, M \in \mathbb{Z}^{+},\left\{f_{n}\right\}_{n=1}^{N},\left\{g_{m}\right\}_{m=1}^{M} \subset H$, we have

$\mathscr{E}_{\phi}^{0}\left(\pi_{\phi}\left(A_{N M}\left(f_{1}, \ldots, f_{N} ; g_{1}, \ldots, g_{M}\right)\right) \mid \mathscr{Y}\right)=\delta_{M N} \operatorname{det}\left\{\mathscr{E}_{\phi}^{0}\left(\pi_{\phi}\left(A_{11}\left(f_{n} ; g_{m}\right)\right) \mid \mathscr{Y}\right)\right\}$.

6) $E_{\phi}^{0}\left(\pi_{\phi}(\mathscr{A}) \mid \mathscr{Y}\right) \cong D_{\phi}(\mathscr{Y})$.

Proof. Since $\mathscr{A}$ is the closed linear span of the $n$-point operators $\left\{A_{N M}\right\}$, existence of $\mathscr{E}_{\phi}^{0}(\mid \mathscr{Y})$ follows by linearity and by continuity (3.7) from Lemma 3.8: uniqueness is immediate. 0 ), 1), 2), and 3) follow from the properties of the conditional expectations $\left\{\mathscr{E}_{\phi}(\mid \tilde{E})\right\}$. It follows from the $G(\tilde{E})$-invariance of $\mathscr{E}_{\phi}(\mid \tilde{E})$ for $\tilde{E} \in \Gamma(\mathscr{Y})$ that 4), holds for $g \in G^{0}(\mathscr{Y}) \equiv \bigcup_{\Gamma(\mathscr{Y})} G(\tilde{E})$. Since $G^{0}(\mathscr{Y})$ is norm-dense in $G(\mathscr{Y})$ (by trivial modification of the proof of $[13,1.3 .1]$ ), and since $\alpha$ and $\mathscr{U}_{\phi}$ are strongly continuous, 4) holds for all $g \in G(\mathscr{Y})$. 5) follows from 3.8, and 6) from 5).

3.12. Proposition. There exists a unique normal conditional expectation $\mathscr{E}_{\phi}(\mid \mathscr{Y})$ on $\pi_{\phi}(\mathscr{A})^{\prime \prime}$ which extends $E_{\phi}^{0}(\mid \mathscr{Y})$. Further, $\mathscr{E}_{\phi}\left(\pi_{\phi}(\mathscr{A})^{\prime \prime} \mid \mathscr{Y}\right)=D_{\phi}(\mathscr{Y})^{\prime \prime}$.

Proof. We first prove existence of $a$, necessarily unique, normal linear map extending $\mathscr{E}_{\phi}^{0}(\mid \mathscr{Y})$. To this end, let $H_{\phi}^{A} \equiv\left\{\pi_{\phi}\left(\mathscr{A}\left(Y_{A} H\right)\right) \Phi\right\}^{-}$, and let $M_{\phi} H_{\phi} \equiv$ $\left[D_{\phi}\left(\mathscr{Y}_{N}\right) H_{\phi}^{A}\right]^{-}$. Since $M_{\phi} \in D_{\phi}(\mathscr{Y})^{\prime}$, and since $\pi_{\phi}\left(\mathscr{A}\left(Y_{N} H\right)\right) \subset D_{\phi}(\mathscr{Y})^{\prime}$ [recalling that $\left.G_{\phi}\left(\mathscr{Y}_{N}\right) \subseteq \mathscr{Z}_{\phi}\right]$, the central support of $M_{\phi}$ in $G_{\phi}(\mathscr{Y})^{\prime \prime}$ is $\mathbb{1}$ (i.e. $\left[D_{\phi}(\mathscr{Y})^{\prime} M_{\phi} H_{\phi}\right]^{-} \supseteqq$ $\left[\pi_{\phi}\left(\mathscr{A}\left(Y_{N} H\right)\right) D_{\phi}\left(\mathscr{Y}_{N}\right) \pi_{\phi}\left(\mathscr{A}\left(Y_{A} H\right)\right) \Phi\right]^{-} \supseteqq\left[\pi_{\phi}(\mathscr{A}(H) \Phi]^{-}=H_{\phi}\right)$. Therefore, the restriction map

$R: D_{\phi}(\mathscr{Y})^{\prime \prime} \rightarrow D_{\phi}(\mathscr{Y})^{\prime \prime} M_{\phi}$ is an isomorphism [4;1, $\S 2$, Proposition 2]. On the other hand, if $S \in \mathscr{A}$, we have

$\left.\mathscr{U}_{\phi}(g) \mathscr{E}_{\phi}\left(\pi_{\phi}(S) \mid \tilde{E}^{0}\right) \mathscr{U}_{\phi}(g)^{*}=\mathscr{U}_{\phi}\left(1+Y_{N}(g-1)\right) \mathscr{E}_{\phi}\left(\pi_{\phi}(S)\right) \mid \tilde{E}^{0}\right) \mathscr{U}_{\phi}\left(1+Y_{N}(g-1)\right)^{*}$

$\forall g \in G$.

Further, since $\mathscr{U}_{\phi}\left(G\left(Y_{N}\right)\right) \subseteq\left\{D_{\phi}\left(\mathscr{Y}_{N}\right) \pi_{\phi}\left(\mathscr{A}\left(Y_{A} H\right)\right)\right\}^{\prime}$, it follows that $\mathscr{U}_{\phi}(g) M_{\phi}=$ $M_{\phi} \forall g \in G\left(Y_{N}\right)$. Combining these two observations, we have

$$
M_{\phi} \mathscr{U}_{\phi}(g) \mathscr{E}_{\phi}\left(\pi_{\phi}(S) \mid \tilde{E}^{0}\right) \mathscr{U}_{\phi}(g)^{*} M_{\phi}=M_{\phi} \mathscr{E}_{\phi}\left(\pi_{\phi}(S) \mid \tilde{E}^{0}\right) M_{\phi} \forall g \in G(\mathscr{Y}) .
$$

Recalling $0.2(3)$, it follows from linearity and continuity that

$$
M_{\phi} \mathscr{E}_{\phi}\left(\mathscr{E}_{\phi}\left(\pi_{\phi}(S) \mid \tilde{E}^{0}\right) \mid \tilde{C}\right) M_{\phi}=M_{\phi} \mathscr{E}_{\phi}\left(\pi_{\phi}(S) \mid \tilde{E}^{0}\right) M_{\phi} \forall \tilde{C} \in \Gamma(\mathscr{Y}) .
$$

Therefore (2.7(4)),

$$
M_{\phi} \mathscr{E}_{\phi}^{0}\left(\pi_{\phi}(S) \mid \mathscr{Y}\right) M_{\phi}=M_{\phi^{\prime}} \mathscr{E}_{\phi}\left(\pi_{\phi}(S) \mid \tilde{E}^{0}\right) M_{\phi} \forall S \in \mathscr{A} .
$$

This proves that $\mathscr{E}_{\phi}^{0}\left(\pi_{\phi}(S) \mid \mathscr{Y}\right)=R^{-1}\left(M_{\phi} \mathscr{E}_{\phi}\left(\pi_{\phi}(S) \mid \tilde{E}^{0}\right) M_{\phi}\right) \forall S \in \mathscr{A}$. Now, since the mapping $S\left(\in \pi_{\phi}(\mathscr{A})^{\prime \prime}\right) \rightarrow M_{\phi^{\circ}} \mathscr{E}_{\phi}\left(S \mid \tilde{E}^{0}\right) M_{\phi}$ is ultra-weakly continuous it follows that $M_{\phi} \mathscr{E}_{\phi}\left(S \mid \tilde{E}^{0}\right) M_{\phi} \in D_{\phi}(\mathscr{Y})^{\prime \prime} M_{\phi} \forall S \in \pi_{\phi}(\mathscr{A})^{\prime \prime}$. We define $\mathscr{E}_{\phi}(\mid \mathscr{Y}) \equiv R^{-1}\left(M_{\phi} \mathscr{E}_{\phi}\left(\mid \tilde{E}^{0}\right) M_{\phi}\right)$ 
on $\pi_{\phi}(\mathscr{A})^{\prime \prime} . \mathscr{E}_{\phi}(\mid \mathscr{Y})$ is clearly a normal linear map extending $\mathscr{E}_{\phi}^{0}(\mid \mathscr{Y})$. To prove $\mathscr{E}_{\phi}(\mid \mathscr{Y})$ is a conditional expectation only (3) of Definition 0.1 remains.

We have,

$$
\begin{aligned}
\mathscr{E}_{\phi}\left(S \mathscr{E}_{\phi}(T \mid \mathscr{Y}) \mid \mathscr{Y}\right) & =R^{-1}\left(M_{\phi} \mathscr{E}_{\phi}\left(S \mathscr{E}_{\phi}(T \mid \mathscr{Y}) \mid \tilde{E}^{0}\right) M_{\phi}\right) \\
& =R^{-1}\left(M_{\phi} \mathscr{E}_{\phi}\left(S \mid \tilde{E}^{0}\right) M_{\phi} \mathscr{E}_{\phi}(T \mid \mathscr{Y}) M_{\phi}\right. \\
& =R^{-1}\left(M_{\phi} \mathscr{E}_{\phi}(S \mid \mathscr{Y}) \mathscr{E}_{\phi}(T \mid \mathscr{Y}) M_{\phi}\right) \\
& =\mathscr{E}_{\phi}(S \mid \mathscr{Y}) \mathscr{E}_{\phi}(T \mid \mathscr{Y}) \quad \forall S, T \in \pi_{\phi}(\mathscr{A})^{\prime \prime}
\end{aligned}
$$

where the second equality follows from $G\left(E^{0}\right)$-invariance $\mathscr{E}_{\phi}(T \mid \mathscr{Y})$. It is clear, from 3.11 and normality, that $E_{\phi}\left(\pi_{\phi}(\mathscr{A})^{\prime \prime} \mid \mathscr{Y}\right) \subseteq D_{\phi}(\mathscr{Y})^{\prime \prime}$. On the other hand, if $S \in D_{\phi}(\mathscr{Y})^{\prime \prime}$, we have

$$
\mathscr{E}_{\phi}(S \mid \mathscr{Y})=R^{-1}\left(M_{\phi} \mathscr{E}_{\phi}\left(S \mid \tilde{E}^{0}\right) M_{\phi}\right)=R^{-1}\left(S M_{\phi}\right)=S .
$$

Hence

$$
\mathscr{E}_{\phi}\left(\pi_{\phi}(\mathscr{A})^{\prime \prime} \mid \mathscr{Y}\right)=D_{\phi}(\mathscr{Y})^{\prime \prime} .
$$

The proof of Theorem 3.2 is immediate from Propositions 3.11 and 3.12. We shall need the following modification of 3.2 .

3.13. Proposition. Let $\left\{\tilde{E}^{n}\right\}_{n=1}^{\infty}$ be a dense, increasing sequence of $\mathscr{Y}$-partitions of $H$; let $\phi$ be a $G(\mathscr{Y})$-invariant state on $\mathscr{A}$. Then,

$$
\sigma-\lim _{n \rightarrow \infty} \mathscr{E}_{\phi}\left(\pi_{\phi}(S) \mid \tilde{E}^{n}\right)=\mathscr{E}_{\phi}\left(\pi_{\phi}(S) \mid \mathscr{Y}\right) \quad \forall S \in \mathscr{A} .
$$

Proof. Let $\tilde{F}=\left\{F_{j}\right\}_{j=1}^{\infty} \geqq E^{0}$ be a $\mathscr{Y}$-partition of $H$ ordered so that $\left.\left\{\Omega, Y_{N} F_{j} \Omega\right)\right\}$ is decreasing. For every $\varepsilon_{1}>0$ there exists $J\left(\varepsilon_{1}\right)$ (finite) such that $\left(\Omega, Y_{N}(1-\right.$ $\left.\left.\sum_{j=1}^{J} F_{j}\right) \Omega\right)<\varepsilon_{1}$. Furthermore, since $\left\{\tilde{E}^{n}\right\}_{n=1}^{N}$ is dense, we have for arbitrary $\varepsilon_{2}$, an $n_{0}$ such that, for each $1 \leqq j \leqq J$, there exists a projector $G_{j}^{\prime}$ which is the sum of orthogonal projectors of $\tilde{E}^{n_{0}}$ and which satisfies $\left(\Omega, G_{j}^{\prime}\left(\mathbb{1}-F_{j}\right)+F_{j}\left(\mathbb{1}-G_{j}^{\prime}\right) \Omega\right)<\varepsilon_{2}$. Let, for each $1 \leqq j \leqq J, G_{j} \equiv G_{j}^{\prime}\left(1-\bigvee_{i<j} G_{i}^{\prime}\right)$; then (1) $G_{j} \leqq G_{j}^{\prime}$, (2) $G_{j} G_{i}=0 \forall i \neq j$, (3) $\sum_{j} G_{j}=\bigvee_{j} G_{j}^{\prime}$, and (4) $G_{j}$ is the sum of orthogonal projectors of $E^{n_{0}}$. It follows directly that

$$
\left(\Omega, F_{j}\left(\mathbb{1}-G_{j}\right)+G_{j}\left(\mathbb{1}-F_{j}\right) \Omega\right)<\varepsilon_{2} J \quad 1 \leqq j \leqq J .
$$

For arbitrary $n \geqq n_{0}$, and projector $D \in \mathscr{Y}$, we have

$$
\begin{aligned}
& \left\|\mathscr{E}\left(N(d) \mid \tilde{F} \bigvee \tilde{E}^{n}\right)-\mathscr{E}\left(N(d) \mid \tilde{E}^{n}\right)\right\|=\| \sum_{j<J}\left\{\mathscr{E}\left(N\left(F_{j} d\right) \mid \tilde{E}^{n}\right)\right. \\
& \left.-\mathscr{E}\left(N\left(G_{j} d\right) \mid \tilde{E}^{n}\right)\right\}+\mathscr{E}\left(N\left(\left(\mathbb{1}-\sum_{j \leqq J} F_{j}\right) d\right) \mid \tilde{E}^{n} \bigvee \tilde{F}\right) \\
& -\mathscr{E}\left(N\left(\left(\mathbb{1}-\sum_{j \leqq J} G_{j}\right) d\right) \mid \tilde{E}^{n}\right)\left\|<\sum_{j \leqq J}\right\| N\left(F_{j} d\right)-N\left(G_{j} d\right) \|+\varepsilon_{1} \\
& +\left(\Omega,\left(\mathbb{1}-\sum_{j \leqq J} G_{j}\right) D \Omega\right) \leqq 2 \varepsilon_{1}+J^{2} \varepsilon_{2}+2 J^{3 / 2}\|\Omega\| \varepsilon_{2}^{1 / 2}
\end{aligned}
$$

To sum up, if $\tilde{F}$ is a $\mathscr{Y}$-partition of $H$, and if $D$ is a projector of $\mathscr{Y}$, then for every $\varepsilon>0$ there exists $n_{0}$ such that $n \geqq n_{0} \Rightarrow$

$$
\left\|\mathscr{E}\left(N(d) \mid \tilde{F} \bigvee \tilde{E}^{n}\right)-\mathscr{E}\left(N(d) \mid \tilde{E}^{n}\right)\right\|<\varepsilon .
$$

The proof is then completed by simple modifications to $3.7,3.8$, and 3.11 . 


\section{3.b Consequences of Local Convergence}

The next lemma aims at establishing (Theorem 3.15) the map $\Omega_{\phi}: \mathscr{Y}_{*} \rightarrow D_{\phi}(\mathscr{Y})^{\prime \prime}$.

3.14. Lemma. Let $\phi$ be a $G(\mathscr{Y})$-invariant state on $\mathscr{A}$. Then, there exists a unique mapping $K_{\phi}:\left(D_{\phi}(\mathscr{Y})^{\prime \prime}\right)_{*} \rightarrow \mathscr{Y}$ such that

$$
\left(g, K_{\phi}(\psi) f\right)=\left\langle\psi ; \mathscr{E}_{\phi}\left(\pi_{\phi}\left(a^{*}(f) a(g)\right) \mid \mathscr{Y}\right)\right\rangle \quad \forall f, g \in H .
$$

Further, $K_{\phi}$ is positive, linear and contracting.

Proof. Since, for each $\psi \in\left(D_{\phi}(\mathscr{Y})^{\prime \prime}\right)_{*}$, the mapping $(g, f) \rightarrow\left\langle\psi ; \mathscr{E}_{\phi}\left(\pi_{\phi}\left(a^{*}(f) a(g)\right) \mid \mathscr{Y}\right)\right\rangle$ is, by Theorem 3.2, a continuous sesquilinear form over $H$, there exists, by Riesz's theorem, a unique bounded linear operator, $K_{\phi}(\psi)$ on $H$ such $\left(g, K_{\phi}(\psi) f\right)=$ $\left\langle\psi ; \mathscr{E}_{\phi}\left(\pi_{\phi}\left(a^{*}(f) a(g)\right) \mid \mathscr{Y}\right)\right\rangle$. By (1) of Theorem 3.2, $h K_{\phi}(\psi) h^{-1}=K_{\phi}(\psi) \forall h \in G(\mathscr{Y})$. It follows that $K_{\phi}(\psi) \in \mathscr{Y}^{\prime}=\mathscr{Y}$. This proves existence and uniqueness. Linearity and positivity are immediate. Finally,

$$
\left\|K_{\phi}(\psi)\right\|=\sup _{\substack{f, g \in H: \\\|f\|=\|g\|=1}}\left|\left\langle\psi ; \mathscr{E}_{\phi}\left(\pi_{\phi}\left(a^{*}(f) a(g)\right) \mid \mathscr{Y}\right)\right\rangle\right| \leqq\|\psi\| .
$$

To each $\Lambda \in \mathscr{Y}_{*}$, we can now assign a mean number operator.

3.15. Theorem. There exists a unique map $\Omega_{\phi}: \mathscr{Y}_{*} \rightarrow D_{\phi}(\mathscr{Y})^{\prime \prime}$ such that

$$
\Omega_{\phi}(\Lambda(g, f))=\mathscr{E}_{\phi}\left(\pi_{\phi}\left(a^{*}(f) a(g)\right) \mid \mathscr{Y}\right) \quad \forall f, g \in H .
$$

Further, $\Omega_{\phi}$ is positive, linear and norm-reducing.

Proof. Uniqueness is immediate. Existence follows from Lemma 3.14 by defining $\Omega_{\phi}(\Lambda)=K_{\phi}^{*}(\Lambda) \forall \Lambda \in \mathscr{Y}_{*}$.

We can now make contact with the discrete case.

3.16. Corollary. Let $A(\mathscr{Y})=\left\{A_{i}\right\}_{i \in I}$ denote the atoms of $\mathscr{Y}$; define for each $i \in I, \Lambda_{i} \in \mathscr{Y}_{*}$ such that $\Lambda_{i}\left(A_{j}\right)=\delta_{i j}$. Then, $\Omega_{\phi}\left(\Lambda_{i}\right)=\pi_{\phi}\left(a^{*}\left(f_{i}\right) a\left(f_{i}\right)\right)$ where $f_{i}$ is any normalized vector in the range of $A_{i}$. Hence $\Omega_{\phi}\left(\Lambda_{i}\right)$ is a projector and $D_{\phi}\left(\mathscr{Y}_{A}\right)$ is generated by $\mathbb{1}_{\phi}$ and $\left\{\Omega_{\phi}\left(\Lambda_{i}\right) \mid i \in I\right\}$.

We now obtain the operator-valued, number density over the spectrum of $\mathscr{Y}$ attached to the representation $\pi_{\phi}$ (cf. $1, \S 7$, Theorems 1 and 2 and III, $\S 3$, Corollary 1 of Ref. [4]).

3.17. Theorem. Let $(X, B, \mu)$ be a totally $\sigma$-finite measure space; and, in the preceding discussion, let $H=\mathscr{L}^{2}(X, B, \mu), \mathscr{Y}=\mathscr{L}^{\infty}(X, B, \mu)$ and $\mathscr{Y}_{*}=\mathscr{L}^{1}(X, B, \mu)$. If $\phi$ is a $G(\mathscr{Y})$-invariant state on $\mathscr{A}(H)$, there exists a $\mu$-a.e. unique, $\sigma$-measurable mapping $N_{\phi}(\mid \mathscr{Y}): X \rightarrow D_{\phi}(\mathscr{Y})^{\prime \prime}$ such that

$$
\left\langle\psi ; \Omega_{\phi}(f)\right\rangle=\int f(x)\left\langle\psi ; N_{\phi}(x \mid \mathscr{Y})\right\rangle d \mu(x) \quad \forall \psi \in\left(D_{\phi}(\mathscr{Y})^{\prime \prime}\right)_{*} ; \forall f \in \mathscr{L}^{1}(X, B, \mu) .
$$

Further, $0 \leqq N_{\phi}(x \mid \mathscr{Y}) \leqq \mathbb{1} \mu$-a.e.

Proof. Separability of $H$ implies that of $\mathscr{A}(H), H_{\phi}$ and hence of $\left(D_{\phi}(\mathscr{Y})^{\prime \prime}\right)_{*}$ and of $\left(D_{\phi}(\mathscr{Y})^{\prime \prime}\right)_{*}^{+}$[the normal positive linear forms on $\left.D_{\phi}(\mathscr{Y})^{\prime \prime}\right]$ : let $D_{*}^{O}$ be a countable dense set of $\left(D_{\phi}(\mathscr{Y})^{\prime \prime}\right)_{*}$ such that $D_{*}^{O} \cap\left(D_{\phi}(\mathscr{Y})^{\prime \prime}\right)_{*}^{+}$in dense in $\left(D_{\phi}(\mathscr{Y})^{\prime \prime}\right)_{*}^{+}$, and let $D_{*}^{O}$ denote the set of all finite linear combinations of elements of $D_{*}^{O}$ with coefficients 
in $Q+i Q(Q=$ rationals $)$. It is clear that $D_{*}^{O}$ is itself a countable dense set of $\left\{D_{\phi}(\mathscr{Y})^{\prime \prime}\right)_{*}$ such that $\left\{D_{*}^{O} \cap\left(D_{\phi}(\mathscr{Y})^{\prime \prime}\right)_{*}^{+}\right\}^{-}=\left(D_{\phi}(\mathscr{Y})^{\prime \prime}\right)_{*}^{+}$. We now prove uniqueness. Suppose that $\bar{N}_{\phi}(\mid \mathscr{Y}), N_{\phi}(\mid \mathscr{Y})$ are two such mappings. It follows from the uniqueness of Lemma 3.14 that for each $\psi \in\left(D_{\phi}(\mathscr{Y})^{\prime \prime}\right)_{*}, \quad K_{\phi}(\psi)_{x}=\left\langle\psi ; N_{\phi}(x \mid \mathscr{Y})\right\rangle=\left\langle\psi ; \bar{N}_{\phi}(x \mid \mathscr{Y})\right\rangle \mu$-a.e. There exists, then, a measurable set $X_{0} \subset X$ such that $\mu\left(X_{0}\right)=0$ and such that $X \bar{\epsilon} U_{0} \Rightarrow$

$$
\left\langle\psi ; N_{\phi}(x \mid \mathscr{Y})\right\rangle=\left\langle\psi ; \bar{N}_{\phi}(x \mid \mathscr{Y})\right\rangle \forall \psi \in D_{*}^{o} .
$$

Since $D_{*}^{O}$ is dense in $\left(D_{\phi}(\mathscr{Y})^{\prime \prime}\right)_{*}$, it follows that $x \bar{\in} X_{0} \Rightarrow N_{\phi}(x \mid \mathscr{Y})=\bar{N}_{\phi}(x \mid \mathscr{Y})$. This proves essential uniqueness of $N_{\phi}(\mid \mathscr{Y})$. We now prove existence. There exists by Lemma 3.14 for each $\psi \in\left(D_{\phi}(\mathscr{Y})^{\prime \prime}\right)_{*}$ a $\mu$-a.e. unique essentially bounded function $K_{\phi}(\psi)$ such that

$$
\left\langle\psi ; \Omega_{\phi}(f)\right\rangle=\int_{X} f(x) K_{\phi}(\psi)_{x} d \mu(x) \forall f \in \mathscr{L}^{1}(X, B, \mu) .
$$

We have, for $\lambda, \gamma \in \mathbb{R}$ and $\psi, \psi^{\prime} \in\left(D_{\phi}(\mathscr{Y})^{\prime \prime}\right)_{*}$.

(1) $K\left(\lambda \psi+\gamma \psi^{\prime}\right)_{x}=\lambda K_{\phi}(\psi)_{x}+\gamma K_{\phi}\left(\psi^{\prime}\right)_{x} \mu$-a.e.

(2) $\left|K_{\phi}(\psi)_{x}\right| \leqq\|\psi\| \mu$-a.e.

(3) $\psi \geqq 0 \Rightarrow K_{\phi}(\psi)_{x} \geqq 0 \mu$-a.e.

There exists, then, a measurable set $X_{0} \subset X$ such that $\mu\left(X_{0}\right)=0$ and such that (1), (2), and (3) hold everywhere on $X-X_{0}$ for $\gamma, \lambda \in Q+i Q$ and for all $\psi, \psi^{\prime} \in D_{*}^{O}$. Define $\bar{K}_{\phi}(\psi)_{x}=K_{\phi}(\psi)_{x} X\left(X-X_{0}\right)$ where $X\left(X-X_{0}\right)$ is the characteristic function of $X-X_{0} \cdot \bar{K}_{\phi}$, mapping $D_{*}^{O}$ into the Banach space of bounded $B$-measurable functions $M(X, B)$, extends by continuity to a complex-linear mapping $\hat{K}_{\phi}$ of $\left(D_{\phi}(\mathscr{Y})^{\prime \prime}\right)_{*}$ into $M(X, B)$. Thus, for each $x \in X, \psi \rightarrow \hat{K}_{\phi}(\psi)_{x}$ is a positive, linear mapping of $\left(D_{\phi}(\mathscr{Y})^{\prime \prime}\right)_{*}$ into $\mathbb{C}$ bounded by 1 . There exists therefore, for each $x \in X$, a unique operator $N_{\phi}(x \mid \mathscr{Y}) \in D_{\phi}(\mathscr{Y})^{\prime \prime}$ such that $\left\langle\psi ; N_{\phi}(x \mid \mathscr{Y})\right\rangle=\hat{K}_{\phi}(\psi)_{x} \forall \psi \in\left(D_{\phi}(\mathscr{Y})^{\prime \prime}\right)_{*}$. One easily verifies that $N_{\phi}(\mid \mathscr{Y})$ satisfies the requirements of the theorem.

3.18. Theorem. Let $\phi$ be a $G(\mathscr{Y})$-invariant state on $\mathscr{A}$, and let $P_{\phi}^{G(\mathscr{Y})}$ denote the cone of $G(\mathscr{Y})$-invariant normal positive linear forms on $\pi_{\phi}(\mathscr{A})^{\prime \prime}$. Then, with the above notation and definitions, the restriction map $R: P_{\phi}^{G(\mathscr{Y})} \rightarrow\left(D_{\phi}(\mathscr{Y})^{\prime \prime}\right)_{*}^{+}$of $P_{\phi}^{G(\mathscr{Y})}$ into the cone of normal positive linear forms on $D_{\phi}(\mathscr{Y})^{\prime \prime}$ is a bijection.

Proof. Let $\tilde{\psi}$ be a normal positive linear form on $D_{\phi}(\mathscr{Y})^{\prime \prime}$. The form $\psi$ defined for each $S \in \pi_{\phi}(\mathscr{A})^{\prime \prime}$ by $\langle\psi ; S\rangle=\left\langle\tilde{\psi} ; \mathscr{E}_{\phi}(S \mid \mathscr{Y})\right\rangle$ is clearly a $G(\mathscr{Y})$-invariant normal positive linear form on $\pi_{\phi}(\mathscr{A})^{\prime \prime}$ extending $\tilde{\psi} . R$ is therefore surjective. Now let $\psi \neq \psi^{\prime} \in P_{\phi}^{G(\mathscr{Y})}$ with $R_{\psi}=R_{\psi^{\prime}}$. Then, there exists $S \in \mathscr{A}$ such that $0 \neq\left\langle\psi-\psi^{\prime} ; \pi_{\phi}(S)\right\rangle$. This, however, results in the contradiction:

$$
\begin{aligned}
0 & =\left\langle R_{\psi}-R_{\psi^{\prime}} ; \mathscr{E}_{\phi}\left(\pi_{\phi}(S) \mid \mathscr{Y}\right)\right\rangle=\left\langle\psi-\psi^{\prime} ; \mathscr{E}_{\phi}\left(\pi_{\phi}(S) \mid \mathscr{Y}\right)\right\rangle \\
& =\lim _{\Gamma(\mathscr{Y})}\left\langle\psi-\psi^{\prime} ; \mathscr{E}_{\phi}\left(\pi_{\phi}(S) \mid \tilde{E}\right)\right\rangle=\left\langle\psi-\psi^{\prime} ; \pi_{\phi}(S)\right\rangle .
\end{aligned}
$$

$R$ is therefore injective.

3.19. Remark. It follows from the proof of 3.18 that if $\phi$ is a $G(\mathscr{Y})$-invariant state on $\mathscr{A}$, and if $\psi$ is a $G(\mathscr{Y})$-invariant normal positive linear form on $\pi_{\phi}(\mathscr{A})^{\prime \prime}$, then $\left\langle\psi ; \mathscr{E}_{\phi}\left(\pi_{\phi}(S) \mid \mathscr{Y}\right)\right\rangle=\left\langle\psi ; \pi_{\phi}(S)\right\rangle \forall S \in \mathscr{A}$. 


\section{Global Theory}

In the present section, we focus on the global consistency of the local results obtained in the preceding section. We first show (4.2) that the set of all $G(\mathscr{Y})$ invariant states on $\mathscr{A}$ is a simplex and give a characterization of the set of extreme points, showing it to be weak* compact. The set $S(\mathscr{Y})$ of extremal $G(\mathscr{Y})$-invariant states supports the global extension we are looking for (4.3-4.5).

The next lemma follows easily from Moore's theorem.

4.0. Lemma. Let $A \in B(H)$ be such that $0 \leqq A \leqq \mathbb{1}$. Let $\omega_{A}$ denote the unique gauge invariant generalized free state on $\mathscr{A}$ such that $\left\langle\omega_{A} ; a^{*}(f) a(g)\right\rangle=(g, A f) \forall$ $f, g \in H$. Then, the map $\omega: B(H)_{1}^{+} \rightarrow F(H)$ is a homeomorphism when the former is equipped with the $\sigma$-topology, and the latter with the weak ${ }^{*}$-topology.

4.1. Definition. Let $\mathscr{Y}$ be a maximal abelian von Neumann algebra on $H$. Let $\bar{S}(\mathscr{Y})$ denote the set of all $A \in \mathscr{Y}$ satisfying $0 \leqq A \leqq \mathbb{1}$ and $A^{2} Y_{A}=A Y_{A}$. Let $S(\mathscr{Y}) \equiv$ $\omega(\bar{S}(\mathscr{Y}))$. We remark that $\bar{S}(\mathscr{Y})$ is a $\sigma$-compact subset of $\mathscr{Y}$; therefore $S(\mathscr{Y})$ is $w^{*}$ compact.

4.2. Theorem. The set $\mathfrak{S}^{G(\mathscr{Y})}$ of all $G(\mathscr{Y})$-invariant states on $\mathscr{A}$ is a (Choquet) simplex, and $S(\mathscr{Y})$ is the set of extremal $G(\mathscr{Y})$-invariant states on $\mathscr{A}$. For each $G(\mathscr{Y})$ invariant state $\phi$ on $\mathscr{A}$, there exists, by Choquet's theorem [cf. 12, A.5], a unique regular probability measure $\mu_{\phi}$ on $\mathfrak{S}^{G(\mathscr{y})}$ (or $\left.\mathfrak{S}\right)$ such that

(1) $\mu_{\phi}(S(\mathscr{Y}))=1$.

(2) $\langle\phi ; A\rangle=\int\langle\sigma ; A\rangle d \mu_{\phi}(\sigma) \quad \forall A \in \mathscr{A}$.

Proof. We show that the cone $P^{G(\mathscr{Y})}$ of $G(\mathscr{Y})$-invariant positive linear forms on $\mathscr{A}$ is a lattice. For this it is sufficient to show that, for each $G(\mathscr{Y})$-invariant state $\phi$, the cone $P_{\phi}^{G(\mathscr{Y})}$ of all $G(\mathscr{Y})$-invariant normal positive linear forms on $\pi_{\phi}(\mathscr{A})^{\prime \prime}$ is a lattice. Indeed, if $\psi, \psi^{\prime} \in P^{G(\mathscr{G})}$, then $\psi, \psi^{\prime} \in P_{\phi}^{G(\mathscr{G})}$ for $\phi=\left(\psi+\psi^{\prime}\right) /\langle\psi+$ $\left.\psi^{\prime} ; \mathbb{1}\right\rangle$. Since $P_{\phi}^{G(\mathscr{G})}$ is, by Theorem 3.18 , order isomorphic to the set of normal positive linear forms on the abelian von Neumann algebra $D_{\phi}(\mathscr{Y})^{\prime \prime}$, and since this last set is a lattice, it follows that $P_{\phi}^{G(\mathscr{y})}$ is a lattice. Hence, $\widetilde{S}^{G(\mathscr{y})}$ is a simplex. We now characterize the set of extremal $G(\mathscr{Y})$-invariant states. Since $P_{\phi}^{G(\mathscr{Q})}$ is order isomorphic to $\left(D_{\phi}(\mathscr{Y})^{\prime \prime}\right)_{*}^{+}$, a $G(\mathscr{Y})$-invariant state $\phi$ on $\mathscr{A}$ is extremal $G(\mathscr{Y})$ invariant if and only if $\left.\phi\right|_{D_{\phi}(\mathscr{G})^{\prime \prime}}$ is a character (the distinction between $\phi$ as a state on $\mathscr{A}$ and its extension to a normal state on $\pi_{\phi}(\mathscr{A})^{\prime \prime}$ is not made explicit). It follows from Theorem 3.2 and Remark 3.19 that if $\left.\phi\right|_{D_{\phi}(\mathscr{O})^{\prime \prime}}$ is a character, then $\phi$ is a gauge invariant generalized free state. Since every gauge invariant generalized free state is a factor $[11,5.1]$, it follows from Lemma 3.10 that $D_{\phi}\left(\mathscr{Y}_{A}\right)^{\prime \prime}=D_{\phi}(\mathscr{Y})^{\prime \prime}$. Now, if $\left.\phi\right|_{D_{\phi}(\mathscr{g})^{\prime \prime}}$ is a character, we have (with the notation of Corollary 3.16),

$$
\begin{aligned}
\left(f_{i}, \omega^{-1}(\phi) f_{i}\right) & =\left\langle\phi ; \pi_{\phi}\left(a^{*}\left(f_{i}\right) a\left(f_{i}\right)\right)\right\rangle=\left\langle\phi ; \pi_{\phi}\left(a^{*}\left(f_{i}\right) a\left(f_{i}\right)\right)^{2}\right\rangle \\
& =\left(f_{i}, \omega^{-1}(\phi) f_{i}\right)^{2}=0 \text { or } 1 .
\end{aligned}
$$

Thus, $Y_{A} \omega^{-1}(\phi)=\Sigma \lambda_{i} A_{i}$ with $\lambda_{i}=0$ or 1 , proving that $Y_{A} \omega^{-1}(\phi)$ is a projector. Conversely if $T \in \mathscr{Y}_{1}^{+}$we have: $\omega_{T}$ is $G(\mathscr{Y})$-invariant, $D_{\omega_{T}}(\mathscr{Y})^{\prime \prime}=D_{\omega_{T}}\left(\mathscr{Y}_{A}\right)^{\prime \prime}$ and, for $i \neq j$,

$$
\begin{aligned}
\left\langle\omega_{T} ; \pi_{\omega_{T}}\left(a^{*}\left(f_{i}\right) a\left(f_{i}\right) a^{*}\left(f_{j}\right) a\left(f_{j}\right)\right)\right\rangle= & \left\langle\omega_{T} ; \pi_{\omega_{T}}\left(a^{*}\left(f_{i}\right) a\left(f_{i}\right)\right)\right\rangle \\
& \cdot\left\langle\omega_{T} ; \pi_{\omega_{T}}\left(a^{*}\left(f_{j}\right) a\left(f_{j}\right)\right)\right\rangle .
\end{aligned}
$$


If, further, $Y_{A} T$ is a projector we have, by reversing the above argument, that

$$
\left\langle\omega_{T} ; \pi_{\omega_{T}}\left(a^{*}\left(f_{i}\right) a\left(f_{i}\right)\right)\right\rangle^{2}=\left\langle\omega_{T} ; \pi_{\omega_{T}}\left(a^{*}\left(f_{i}\right) a\left(f_{i}\right)\right)^{2}\right\rangle \quad \forall i \in I .
$$

Since $D_{\omega_{T}}\left(\mathscr{Y}_{A}\right)^{\prime \prime}=\left\{\pi_{\omega_{T}}\left(a^{*}\left(f_{i}\right) a\left(f_{i}\right)\right)\right\}_{i \in I}^{\prime \prime}, \omega_{T}$ is a character on $D_{\omega_{T}}(\mathscr{Y})^{\prime \prime}$ and is therefore an extremal $G(\mathscr{Y})$-invariant state on $\mathscr{A}$. Assertions 1 and 2 follow immediately from Choquet's theorem, bearing in mind that $S(\mathscr{Y})$ is compact and $\mathfrak{S}$ is metrizable.

4.3. (a) Definition. Let $D(\mathscr{Y})$ denote the $C^{*}$-algebra of continuous, complexvalued functions on the compact Hausdorff space $S(\mathscr{Y})$. Let $\mathscr{E}(\mid \mathscr{Y})$ denote the canonical map of $\mathscr{A}$ into $D(\mathscr{Y})$. That is, for each $T \in \mathscr{A}, \mathscr{E}(T \mid \mathscr{Y})(\sigma) \equiv\langle\sigma ; T\rangle \forall \sigma \in$ $S(\mathscr{Y})$.

We remark that since (3.19 and 3.15) for $f, g \in H$ and $\sigma \in S(\mathscr{Y})\left\langle\sigma ; a^{*}(f) a(g)\right\rangle=$ $\left\langle\sigma ; \Omega_{\sigma}(\Lambda(g, f))\right\rangle$, and since $\mathscr{Y}_{*}$ consists of vector-forms, we can add:

4.3. (b) Definition. Let $\Omega: \mathscr{Y}_{*} \rightarrow D(\mathscr{Y})$ be defined, for each $\sigma \in S(\mathscr{Y})$ and $\Lambda \in \mathscr{Y}_{*}$, by $\Omega(\Lambda)(\sigma)=\left\langle\sigma ; \Omega_{\sigma}(\Lambda)\right\rangle$.

4.4. Remark. It is clear that $\mathscr{E}(\mid \mathscr{Y})$ is a positive, *-preserving, linear map of norm 1: we show in 4.5 that it is "almost" a conditional expectation.

It is also clear that $\Omega: \mathscr{Y}_{*} \rightarrow D(\mathscr{Y})$ is a positive, linear, contractive injection which satisfies the formula:

$$
\mathscr{E}\left(A_{N M}\left(f_{1}, \ldots, f_{N} ; g_{1}, \ldots, g_{M}\right) \mid \mathscr{Y}\right)=\delta_{M N} \operatorname{det}\left\{\Omega\left(\Lambda\left(g_{m}, f_{n}\right)\right)\right\} \text {. }
$$

4.5. Theorem. Let $\phi$ be a $G(\mathscr{Y})$-invariant state on $\mathscr{A}$. There exists a unique *-representation $\hat{\pi}_{\phi}: D(\mathscr{Y}) \rightarrow B\left(H_{\phi}\right)$ such that

$$
\mathscr{E}_{\phi}\left(\pi_{\phi}(S) \mid \mathscr{Y}\right)=\hat{\pi}_{\phi} \mathscr{E}(S \mid \mathscr{Y}) \quad \forall S \in \mathscr{A} .
$$

Further, $\hat{\pi}_{\phi}(\Omega(\Lambda))=\Omega_{\phi}(\Lambda) \quad \forall \Lambda \in \mathscr{Y}_{*}$.

Proof. Uniqueness would follow by continuity from norm density of $\mathscr{E}(\mathscr{A} \mid \mathscr{Y})$ in $D(\mathscr{Y})$. Since $\mathscr{E}(\mathscr{A} \mid \mathscr{Y})$ is a self-adjoint, linear subspace of $D(\mathscr{Y})$, separating $S(\mathscr{Y})$ and containing the unit, density will follow from the Stone-Weierstrass theorem if $\mathscr{E}(\mathscr{A} \mid \mathscr{Y})^{-N}$ can be proven to be an algebra. It is sufficient to show that $U, V \in \mathscr{A} \Rightarrow$ $\mathscr{E}(V \mid \mathscr{Y}) \mathscr{E}(U \mid \mathscr{Y}) \in \mathscr{E}(\mathscr{A} \mid \mathscr{Y})^{-N}$. We have, for arbitrary $\sigma \in S(\mathscr{Y})$,

$$
\begin{aligned}
& \mathscr{E}(V \mid \mathscr{Y}) \cdot \mathscr{E}(U \mid \mathscr{Y})(\sigma) \\
& =\left\langle\sigma ; \mathscr{E}_{\sigma}\left(\pi_{\sigma}(V) \mid \mathscr{Y}\right) \mathscr{E}_{\sigma}\left(\pi_{\sigma}(U) \mid \mathscr{Y}\right)\right\rangle=\left\langle\sigma ; \pi_{\sigma}(V) \mathscr{E}_{\sigma}\left(\pi_{\sigma}(U) \mid \mathscr{Y}\right)\right\rangle \\
& =\lim _{n \rightarrow \infty}\left\langle\sigma ; V \mathscr{E}\left(U \mid \tilde{E}^{n}\right)\right\rangle=\lim _{n \rightarrow \infty} \mathscr{E}\left(V \mathscr{E}\left(U \mid \tilde{E}^{n}\right) \mid \mathscr{Y}\right)(\sigma),
\end{aligned}
$$

where $\left\{\tilde{E}^{n}\right\}$ is a dense increasing sequence of $\mathscr{Y}$-partitions of $H$ [by 3.2(2), 3.13, 3.19 , and the fact that $\sigma$ is a character on $\left.D_{\sigma}(\mathscr{Y})^{\prime \prime}\right]$.

Consequently, [6, IV.6.4] $\mathscr{E}(V \mid \mathscr{Y}) \mathscr{E}(U \mid \mathscr{Y})=$ weak- $\lim _{n \rightarrow \infty} \mathscr{E}\left(V \mathscr{E}\left(U \mid \tilde{E}^{n}\right) \mid \mathscr{Y}\right)$ (the continuous function on the left is the point-wise limit of a bounded sequence of continuous functions). By $[6, \mathrm{~V} .3 .13] \mathscr{E}(V \mid \mathscr{Y}) \mathscr{E}(U \mid \mathscr{Y}) \in \mathscr{E}(\mathscr{A} \mid \mathscr{Y})^{-N}$.

This completes the proof of uniqueness; we now prove existence. Let $S \in \mathscr{A}$,

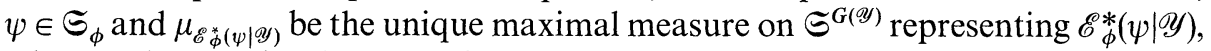
where $\mathscr{E}_{\phi}^{*}(\psi \mid \mathscr{Y})$ is the $G(\mathscr{Y})$-invariant, normal state on $\pi_{\phi}(\mathscr{A})^{\prime \prime}$ defined as $\left\langle\mathscr{E}_{\phi}^{*}(\psi \mid \mathscr{Y}) ; S\right\rangle \equiv\left\langle\psi ; \mathscr{E}_{\phi}(S \mid \mathscr{Y})\right\rangle$. We have

$$
\left\langle\psi ; \mathscr{E}_{\phi}\left(\pi_{\phi}(S) \mid \mathscr{Y}\right)\right\rangle \equiv\left\langle\mathscr{E}_{\phi}^{*}(\psi \mid \mathscr{Y}) ; S\right\rangle=\int \mathscr{E}(S \mid \mathscr{Y})(\sigma) d \mu_{\mathscr{E}_{\phi}^{*}(\psi \mid \mathscr{Y})}(\sigma)
$$


We can now define, without ambiguity, $\hat{\pi}_{\phi}^{0}: \mathscr{E}(\mathscr{A} \mid \mathscr{Y}) \rightarrow B\left(H_{\phi}\right)$ by $\hat{\pi}_{\phi}^{0}(\mathscr{E}(S \mid \mathscr{Y})) \equiv$ $\mathscr{E}_{\phi}\left(\pi_{\phi}(S) \mid \mathscr{Y}\right) \forall S \in \mathscr{A}$. It is clear that $\hat{\pi}_{\phi}^{0}$ is linear and *-preserving. Let again $U, V \in \mathscr{A}$; then, by the weak convergence proven above, we have

$$
\begin{aligned}
& \left\langle\psi ; \mathscr{E}_{\phi}\left(\pi_{\phi}(U) \mid \mathscr{Y}\right) \mathscr{E}_{\phi}\left(\pi_{\phi}(V) \mid \mathscr{Y}\right)\right\rangle=\lim _{n \rightarrow \infty}\left\langle\mathscr{E}_{\phi}^{*}(\psi \mid \mathscr{Y}) ; U \mathscr{E}\left(V \mid \tilde{E}^{n}\right)\right\rangle \\
& =\lim _{n \rightarrow \infty} \int \mathscr{E}\left(U \mathscr{E}\left(V \mid \tilde{E}^{n}\right) \mid \mathscr{Y}\right)(\sigma) d \mu_{\mathscr{E}_{\phi}^{*}(\psi \mid \mathscr{Y})}(\sigma) \\
& =\int \mathscr{E}(U \mid \mathscr{Y}) \mathscr{E}(V \mid \mathscr{Y})(\sigma) d \mu_{\mathscr{E} \Psi(\psi \mid \mathscr{Y})}(\sigma) .
\end{aligned}
$$

Thus, for each $V \in \mathscr{A}$ we have,

$$
\begin{aligned}
& \left\|\mathscr{E}_{\phi}\left(\pi_{\phi}(V) \mid \mathscr{Y}\right)\right\|^{2} \\
& =\sup _{\|\xi\|=1}\left(\xi, \mathscr{E}_{\phi}\left(\pi_{\phi}(V) \mid \mathscr{Y}\right) \mathscr{E}_{\phi}\left(\pi_{\phi}(V) \mid \mathscr{Y}\right) \xi\right) \leqq\|\mathscr{E}(V \mid \mathscr{Y})\|_{\text {sup-norm }}^{2} .
\end{aligned}
$$

Therefore, $\hat{\pi}_{\phi}^{0}$ extends by continuity to a continuous, *-preserving, linear map $\hat{\pi}_{\phi}$ of $D(\mathscr{Y})=\mathscr{E}(\mathscr{A} \mid \mathscr{Y})^{-N}$ into $G_{\phi}(\mathscr{Y})$. It is clear that, for each normal form $\psi$ on $\pi_{\phi}(\mathscr{A})^{\prime \prime},\left\langle\psi ; \hat{\pi}_{\phi}(T)\right\rangle=\int T(\sigma) d \mu_{\mathscr{E} \mathscr{\phi}(\psi \mid \mathscr{Y})}(\sigma) \forall T \in D(\mathscr{Y})$.

Hence, for $U, V \in \mathscr{A}$, we have

$$
\hat{\pi}_{\phi}(\mathscr{E}(U \mid \mathscr{Y}) \mathscr{E}(V \mid \mathscr{Y}))=\hat{\pi}_{\phi}(\mathscr{E}(U \mid \mathscr{Y})) \cdot \hat{\pi}_{\phi}(\mathscr{E}(V \mid \mathscr{Y})) .
$$

Hence, $\hat{\pi}_{\phi}$ is a $*$-representation of $D(\mathscr{Y})$. The remaining assertion is immediate.

4.6. Corollary. Let $\mathfrak{S}(\mathscr{Y})$ denote the set of regular probability measures on $S(\mathscr{Y})$. Then, the map $\mathscr{E}^{*}(\mid \mathscr{Y}): \Im(\mathscr{Y}) \rightarrow \mathfrak{S}^{G(\mathscr{Y})}$ defined for each $\mu \in \mathfrak{S}(\mathscr{Y})$ by

$$
\langle\mathscr{E} *(\mu \mid \mathscr{Y}) ; S\rangle=\int_{\Xi(\mathscr{Y})} \mathscr{E}(S \mid \mathscr{Y})(\sigma) d \mu(\sigma) \forall S \in \mathscr{A}
$$

is an affine, bijective map.

\section{Discussion}

In Theorem 3.2, the net of conditional expectations is proven to converge only on $\pi_{\phi}(\mathscr{A})$ (not on $\left.\pi_{\phi}(\mathscr{A})^{\prime \prime}\right)$. If $\phi$ is the Fock state and if $\mathscr{Y}$ is non-atomic, there exist elements of $\pi_{\phi}(\mathscr{A})^{\prime \prime}$ on which the net does not converge. By restricting our attention to $\pi_{\phi}(\mathscr{A})$, we have been able to treat those cases when $\pi_{\phi}(\mathscr{A})^{\prime \prime}$ is not $G(\mathscr{Y})$-finite.

We remark that Corollary 4.6 is the natural generalization of the work of Shale and Stinespring on states symmetric about a basis [14]. Araki [1] has generalized Theorem 4.2.

On the physical side, we have isolated a classical field of number densities on the spectrum of an arbitrary, complete one-partical observable $\mathcal{O}$. When $\mathcal{O}$ has discrete spectrum, the field is simply the lattice gas [3]. When $\mathcal{O}$ has continuous spectrum, the field is macroscopic (i.e. centre-valued [10]). This results from the coarseness of the CAR-algebra description of a Fermi field on a continuous physical space; coarsness which is preserved by normality of the projector upon the classical field. 


\section{Appendix}

Proof of Theorem 0.2. We give a sketch. See 16.I for details and notation.

1) Existence of the extension follows from amenability of $G$ and the assumption $\mathscr{A}^{M} \cong \mathscr{A}^{G}$ (e.g. Ruelle $[12,6.2 .13]$ ): Uniqueness is immediate.

2) Since each state on $\mathscr{A}^{G}$ can be extended (e.g. Ruelle) to a G-invariant state on $\mathscr{A}, \mathscr{A}^{M}$ separates the set of all states on $\mathscr{A}^{G}$. Therefore, by an Extension of the Stone-Weierstrass theorem $[5,11.3 .1], \mathscr{A}^{M}=\mathscr{A}^{G}$.

3) Let $\mathscr{B}$ be the eneveloping von-Neumann algebra of $\mathscr{A} \cdot \mathscr{A}$ is isomorphic to a $\sigma$-dense $C^{*}$-subalgebra of $\mathscr{B}$, and $G$ extends to a group of automorphisms of $\mathscr{B}$. If $S \in \mathscr{B}$ (resp. $\phi \in \mathscr{B}^{*}$ ), and if $m$ is a mean on $B(G)$, define $m S \in \mathscr{B}$ (resp. $\left.m^{*} \phi \in \mathscr{B}^{*}\right)$ by $\langle m S ; \mu\rangle=m\langle\dot{g} S ; \mu\rangle \forall \mu \in \mathscr{B}_{*}$ (resp. $\left.\left\langle m^{*} \phi ; S\right\rangle=m\langle\phi ; \dot{g} S\rangle \forall S \in \mathscr{B}\right)$. If $\phi$ is a state on $\mathscr{B}$ and if $\eta$ is an invariant mean, then it is easily seen that $\phi \circ \eta$ and $\eta^{*} \phi$ are two $G$-invariant states on $\mathscr{B}$ which coincide on $\mathscr{A}^{G}$. Consequently, by $1,\langle\phi ; \eta S\rangle=\left\langle\eta^{*} \phi ; S\right\rangle \forall S \in \mathscr{A}$ (though not necessarily for all $S \in \mathscr{B}$ ). Choose a net $\left\{M_{\beta}\right\}_{\beta \in I}$ of finite means weak ${ }^{*}$-convergent to $\eta$. Then, for each state $\phi$ on $\mathscr{B}$ and $S \in \mathscr{A},\langle\phi ; \eta S\rangle=\left\langle\eta^{*} \phi ; S\right\rangle=\eta\langle\phi ; \dot{g} S\rangle=\lim M_{\beta}\langle\phi ; \dot{g} S\rangle=\lim \left\langle\phi ; M_{\beta} S\right\rangle$. Thus, by linearity, for each $S \in \mathscr{A}, \eta S=$ weak- $\lim M_{\beta} S$. We conclude that $\eta S \in \operatorname{Co}\{g S \mid g \in G\}^{- \text {weak }}$ and by Mazur's theorem [6, V.3.13], that $\eta S \in \operatorname{Co}\{g S \mid g \in G\}^{-N}$. Consequently, $\operatorname{Co}\{g S \mid g \in G\}^{-N} \cap \mathscr{A}^{G}$ is not empty. To prove that $\{\eta S\}=$ $\operatorname{Co}\{g S \mid g \in G\}^{-N} \cap \mathscr{A}^{G}$ suppose that $\bar{S} \in \operatorname{Co}\{g S \mid g \in G\}^{-N} \cap \mathscr{A}^{G}$ with $\bar{S} \neq \eta S$. There exists, therefore, $\phi \in \mathscr{B}^{*}$ such that $\langle\phi ; \bar{S}-\eta S\rangle \neq 0$; thus $\left\langle\eta^{*} \phi ; \bar{S}-\eta S\right\rangle \neq 0$. But $\eta^{*} \phi$ is, by continuity, and linearity, constant over $\operatorname{Co}\{g S \mid g \in G\}^{-N}$. Define $S^{G}=\eta S$.

4) These properties follow immediately from those of the invariant mean $\eta$. Uniqueness is immediate.

\section{References}

1. Araki,H.: Kyoto preprint

2. Balslev, E., Manuceau, J., Verbeure, A. : Commun. math. Phys. 8, 315-326 (1968)

3. Brascamp, H.J.: Commun. math. Phys. 18, 82-96 (1970)

4. Dixmier, J.: Les Algèbres d'opérateurs dans l'espace Hilbertien. Paris: Gauthier-Villars 1969

5. Dixmier, J.: Les $C^{*}$-algèbres et leurs représentations. Paris: Gauthier-Villars (1969)

6. Dunford, N., Schwartz, J.: Linear operators. I. New York: Interscience 1958

7. Emch, G. G.: Algebraic methods in statistical mechanics and quantum field theory. New York: John Wiley and Sons 1972

8. Halmos, P.: Measure theory. New York: Van Nostrand 1950

9. Kovács, I., Szücs, J.: Szeged Acta. Sci. Math. 27, 233-246 (1966)

10. Lanford III, O. E., Ruelle, D.: Commun. math. Phys. 13, $194-215$ (1969)

11. Powers, R., Stormer, E.: Commun. math. Phys. 16, 1-33 (1970)

12. Ruelle, D.: Statistical mechanics. New York: W. A. Benjamin 1969

13. Sakai, S.: $C^{*}$-algebras and $W^{*}$-algebras. Berlin-Heidelberg-New York: Springer 1971

14. Shale, D., Stinespring, W.F.: Ann. Math. 80, 365-381 (1964)

15. Stormer, E.: Commun. math. Phys. 16, 136-137 (1970)

16. Wolfe, J., Emch, G. G. : JMP 15, 1343 (1974)

Communicated by $\mathrm{H}$. Araki

John C. Wolfe Physics Department University of British Columbia Vancouver, British Columbia V6T 1W5 Canada 